\title{
Whole genome sequencing of Saccharomyces cerevisiae: from genotype to phenotype for improved metabolic engineering applications
}

José Manuel Otero ${ }^{1,2,5+}$, Wanwipa Vongsangnak ${ }^{1,2,8+}$, Mohammad A Asadollahi ${ }^{1,2,6}$, Roberto Olivares-Hernandes ${ }^{1,2}$, Jérôme Maury ${ }^{2,7}$, Laurent Farinelli ${ }^{3}$, Loïc Barlocher ${ }^{3}$, Magne Østerås ${ }^{3}$, Michel Schalk ${ }^{4}$, Anthony Clark ${ }^{4}$, Jens Nielsen ${ }^{1,2^{*}}$

\begin{abstract}
Background: The need for rapid and efficient microbial cell factory design and construction are possible through the enabling technology, metabolic engineering, which is now being facilitated by systems biology approaches. Metabolic engineering is often complimented by directed evolution, where selective pressure is applied to a partially genetically engineered strain to confer a desirable phenotype. The exact genetic modification or resulting genotype that leads to the improved phenotype is often not identified or understood to enable further metabolic engineering.
\end{abstract}

Results: In this work we performed whole genome high-throughput sequencing and annotation can be used to identify single nucleotide polymorphisms (SNPs) between Saccharomyces cerevisiae strains S288C and CEN.PK113-7D. The yeast strain S288C was the first eukaryote sequenced, serving as the reference genome for the Saccharomyces Genome Database, while CEN.PK113-7D is a preferred laboratory strain for industrial biotechnology research. A total of 13,787 high-quality SNPs were detected between both strains (reference strain: S288c). Considering only metabolic genes (782 of 5,596 annotated genes), a total of 219 metabolism specific SNPs are distributed across 158 metabolic genes, with 85 of the SNPs being nonsynonymous (e.g., encoding amino acid modifications). Amongst metabolic SNPs detected, there was pathway enrichment in the galactose uptake pathway (GAL1, GAL10) and ergosterol biosynthetic pathway (ERG8, ERG9). Physiological characterization confirmed a strong deficiency in galactose uptake and metabolism in S288c compared to CEN.PK113-7D, and similarly, ergosterol content in CEN. PK113-7D was significantly higher in both glucose and galactose supplemented cultivations compared to S288c. Furthermore, DNA microarray profiling of S288C and CEN.PK113-7D in both glucose and galactose batch cultures did not provide a clear hypothesis for major phenotypes observed, suggesting that genotype to phenotype correlations are manifested post-transcriptionally or post-translationally either through protein concentration and/or function.

Conclusions: With an intensifying need for microbial cell factories that produce a wide array of target compounds, whole genome high-throughput sequencing and annotation for SNP detection can aid in better reducing and defining the metabolic landscape. This work demonstrates direct correlations between genotype and phenotype that provides clear and high-probability of success metabolic engineering targets. The genome sequence, annotation, and a SNP viewer of CEN.PK113-7D are deposited at http://www.sysbio.se/cenpk.

\footnotetext{
*Correspondence: nielsenj@chalmers.se

† Contributed equally

'Department of Chemical and Biological Engineering, Chalmers University of

Technology, SE-41296 Gothenburg, Sweden

Full list of author information is available at the end of the article
}

\section{Biomed Central}

(c) 2010 Otero et al; licensee BioMed Central Ltd. This is an Open Access article distributed under the terms of the Creative Commons Attribution License (http://creativecommons.org/licenses/by/2.0), which permits unrestricted use, distribution, and reproduction in any medium, provided the original work is properly cited. 


\section{Background}

Metabolic engineering is the enabling technology for identification of targeted genetic modifications such as gene deletion, over-expression, or modulation. The genetic engineering implemented in a host microbial cell factory ideally will lead to re-direction of fluxes to enhance production or robustness of a given product or organism, respectively [1-5]. Metabolic engineering through systems biology has been complimented, and its application expanded in both scope and success. Systems biology is a multi-disciplinary approach to quantitative collection, analysis, and integration of whole genome scale data sets enabling construction of biologically relevant and often predictive mathematical models [6-8]. Genome sequencing of industrially relevant organisms, including S. cerevisiae strain S288c, the first eukaryote genome sequence reported, provided a framework for gene annotation through functional genomics. More relevant to metabolic engineering, an annotated genome sequence was a prerequisite for genome-scale metabolic network reconstructions $[9,10]$. Such reconstructions offer a biochemical model describing the formation and depletion of each metabolite that by providing mass-balance boundary conditions makes possible constraint based simulations of how the metabolic network operates at different conditions. In simpler terms, using basic stoichiometry these models can be used to predict the relationships between gene functions in the cellular metabolic network. With nearly 14 years elapsing since the $S$. cerevisiae strain S288c genome sequence was made available, and more than 1,000 laboratories participating in functional genomics efforts, there are still 968 and 811 open reading frames (ORFs) classified as uncharacterized and dubious, respectively, according to the Saccharomyces Genome Database (SGD) [10,11]. Furthermore, since 2003 there have been published five major S. cerevisiae genome-scale metabolic network reconstructions, with the most recent models encompassing between $13-14 \%$ genome coverage $[12,13]$. The opportunity to further extend genotype to phenotype annotation is abundant.

Industrial biotechnology is dominated by efforts to confer a desirable phenotype onto strains using different methods of directed evolution and random mutagenesis, requiring screening and selection. This approach, while providing little to no mechanistic understanding of which specific genetic perturbations lead to improved strains so they could be further exploited, has proven to be commercially successful as illustrated by the more than 1,000 fold improvement in penicillin titer by Penicillium chrysogenum [14]. As industrial biotechnology applications expand, and the desire to custom-engineer microbial cell factories with novel architecture for native and heterologous metabolic pathways increases, the necessity on a genome-wide level to understand direct genotype to phenotype relationships has rapidly increased. Within the same time period of approximately the last 10 years, the technologies and costs associated with whole genome sequencing have advanced and decreased, respectively. There are several excellent reviews of genome sequencing technologies, and their applications to functional genomics, strain engineering, and other investigatory biology efforts [5,15-18]. Prior work, specifically focused on characterizing genomewide analysis of nucleotide polymorphisms in S. cerevisiae utilized 25 mers oligonucleotide microarrays (Affymetrix yeast tiling arrays) providing random and redundant coverage of the S. cerevisiae genome [19]. This analysis included single nucleotide polymorphism (SNP) identification between S288c and the commonly used laboratory strain S. cerevisiae CEN.PK, where a total of 13,914 SNPs were identified. However, this approach is unable to identify the exact nucleotide substitution, and consequently whether the transcribed SNP results in an amino acid substitution, presumably required to confer a change in enzyme and/or protein function. More recently a collaborative project, the Saccharomyces Genome Resequencing Project (SGRP) between the Sanger Institute and Institute of Genetics, University of Nottingham, completed the ABI sequencing of haploids of 37 S. cerevisiae strains to a coverage of 1-3X. Furthermore, Illumina-Solexa genome sequencing of four of the $37 \mathrm{~S}$. cerevisiae strains, one of which included S288c, was completed [20]. This sequencing effort was focused on exploration of genomic variation in the context of evolution, thereby using multiple strains from different Saccharomyces species. It is a demonstration of a recent genome sequencing technology, referred to as Illumina-Solexa sequencing, compared to larger read methods such as Sanger or 454 sequencing. Illumina-Solexa sequencing is an ultra-highthroughput technology that performs sequencing-bysynthesis of random arrays of clonal DNA colonies attached to the surface of a flow-cell. The approach used in this study generates short, 35 base pair (bp) reads (currently, the technology limitations are $150 \mathrm{bp}$ or $2 \times 150$ bp paired-reads), that must then be aligned to and assembled using a reference genome [21-23].

In this work we propose that high-throughput genome sequencing and annotation, integrated with a genome browser and SNP viewer of S. cerevisiae may serve as a commonplace tool, complementary to transcriptomics and physiological characterization, to extract direct genotype to phenotype information. Firstly, whole genome Illumina-Solexa sequencing of each strain was completed and then annotation was performed. To access annotated genome sequences and detected SNPs of CEN.PK113-7D, a genome browser and SNP viewer 
were developed. SNPs strictly related to metabolic genes were identified, characterized, and amino acid level analysis performed. In order to directly link genotype to phenotype cellular behavior was characterized in wellcontrolled batch fermentations on glucose and galactose, complimented with transcriptome analysis. More specifically, we demonstrate that S288c, the strain utilized for the publically available $S$. cerevisiae genome sequence, exhibits atypical $S$. cerevisiae behavior related to central carbon metabolism as compared to CEN.PK113-7D, a common laboratory strain for industrial biotechnology applications [24].

There were clear correlations between physiology and metabolic pathway enrichment of nonsynonymous SNPs observed, suggesting that sequencing and the annotated genome may assist in reducing the genetic target space for metabolic engineering applications. The analysis presented here serves as a foundation for comparative metabolic engineering SNP analysis, wherein the future reference strains may be compared to their metabolically engineered derivatives that use directed evolution in order to answer the age-old question: what changed in our strain that makes it a preferred microbial cell factory?

\section{Results}

\section{Genome Sequencing and Annotation}

The genome sequencing of $S$. cerevisiae strain CEN. PK113-7D and genome re-sequencing of strain S288c were accomplished using the Illumina/Solexa technology. According to the manufacturer's recommendations (Illumina), raw sequence reads of strain CEN.PK113-7D and strain S288c were accumulated to approximately $18 \times$ and $15 \times$ depth coverage, respectively. Reads were mapped on the S288c as reference genome using MAQ software http://maq.sourceforge.net. Based on genome sequence analysis and annotation (See Methods), the 12.1 $\mathrm{Mb}$ genome was predicted to contain a total of 5,596 genes encoding proteins. The genome was predicted to comprise 16 chromosomes by pair-wise comparison against the 16 different chromosomes of the public reference strain S288c from the Saccharomyces Genome Database (SGD). Interestingly, we found that genome characteristics of strain CEN.PK113-7D are very similar to S288c for genome parameters that include total size, chromosome length, GC content, and the number of predicted genes. Table 1 summarizes Illumina genome sequencing and annotation results.

\section{Genome Browser and SNP Viewer}

To visualize genome sequences, annotated genes, and detected SNPs of $S$. cerevisiae CEN.PK 113-7D, a PHPMySQL-based genome browser and SNP viewer were developed and deposited on the web-site (See http://
Table 1 Illumina/Solexa genome sequencing and annotation results

\begin{tabular}{lcc}
\hline Sequencing Parameter & S288c & CEN.PK113-7D \\
\hline No. of Reads & $5,301,907$ & $6,603,200$ \\
No. of Aligned Reads & $5,176,155$ & $6,216,656$ \\
Total Bases ${ }^{\mathbf{A}}$ (bp) & $181,165,425$ & $217,582,960$ \\
Calculated Average Coverage & $15 x$ & $18 \times$ \\
Genome Percent Reference & 99.9 & 99.4 \\
Coverage (\%) & & \\
No. of chromosomes & 16 & 16 \\
\%GC content & 38.3 & 38.3 \\
\hline
\end{tabular}

Notes: Base pairs (bp). (A) Each read is 35 base pairs in length.

www.sysbio.se/cenpk). The basic genome browser functionality, as seen in Figure 1, can provide genome annotation views with an overhead bar providing a visual indication of chromosome position. It is possible to navigate by dragging the display left or right creating a smooth panning effect. Alternatively, one can navigate directly to a region (e.g., gene name) of interest by providing the region coordinates or typing a feature name into the quick search box. The browser can display basic genomic features of interest (e.g., geneID, gene name, gene function, location, gene ontology process, and exon/intron structures). Similarly, for the SNP viewer one can visualize nucleotide and amino acid polymorphism data between $S$. cerevisiae strains. The whole gene containing a SNP (s) can be displayed by dragging left or right button (which creates a smooth panning effect), and also highlight the mutated position (e.g., nonsynonymous SNP (s), silent SNP (s)) between S. cerevisiae strains.

\section{SNPs identification in metabolism}

Based on whole genome sequencing, data that includes the number of reads, average coverage relative to the SGD reference genome, total number of non-ambiguous SNPs, and total number of filtered SNPs and detected SNPs error are presented in Table 2. Not surprisingly, S288c had relatively few SNPs compared to CEN. PK113-7D given that the reference genome from SGD is based on S288c version 12.0 [10,11]. Furthermore, the 13,787 filtered SNPs identified using the MAQ software is consistent with the previously estimated 13,914 SNPs for CEN.PK113-7D based upon DNA hybridization to 25 mers olignonucleotide microarrays [19]. Table 2 also presents the results for metabolic SNP detection, where a total of 782 metabolic genes as defined by SGD were used to query for SNPs in both the S288c and CEN. PK113-7D genome sequences. A total of 36 metabolic SNPc of S288c, 3 of which are nonsynonymous, were identified across 14 independent metabolic genes (3 nonsynonymous SNPs distributed across 3 metabolic genes). 


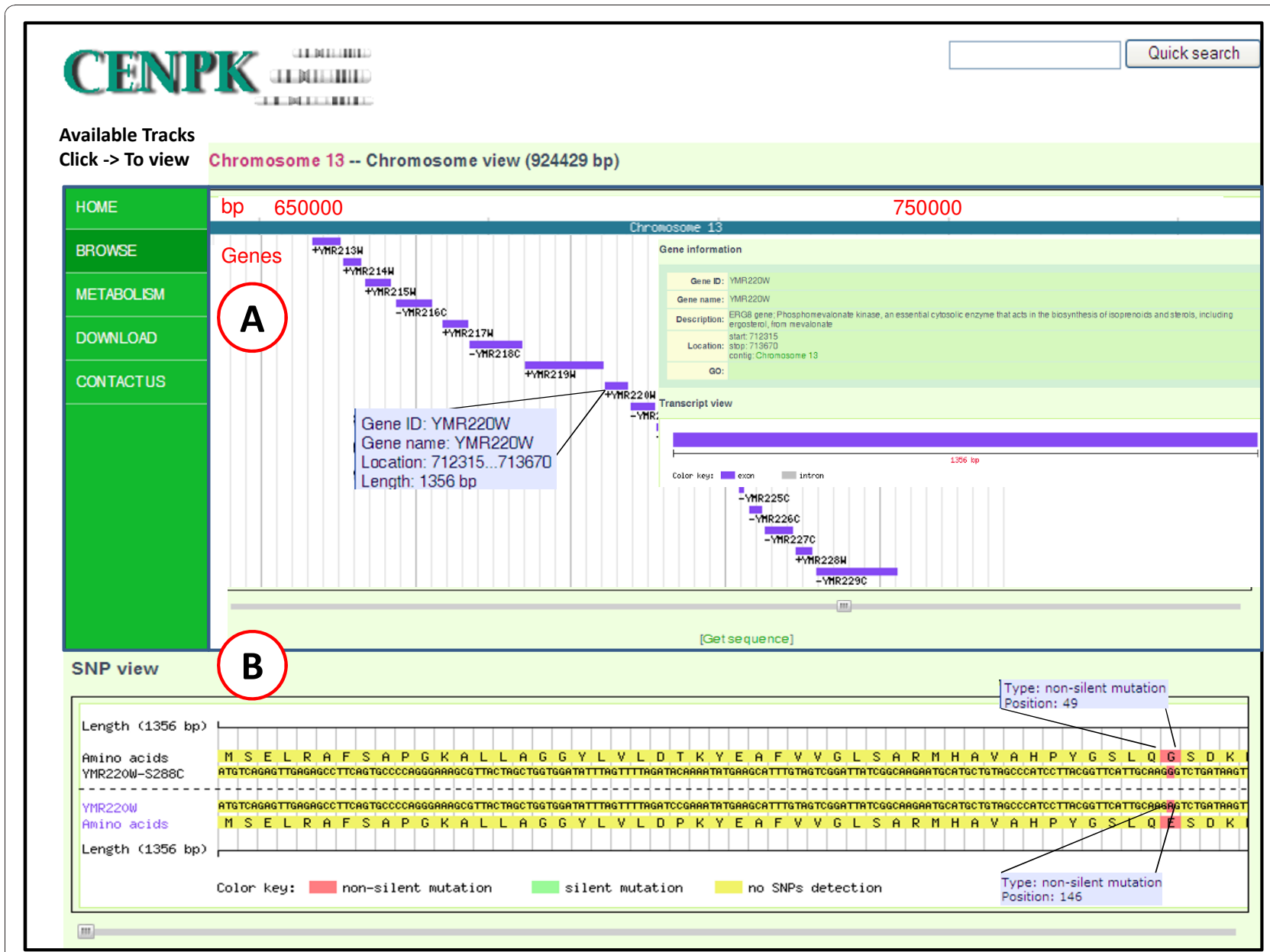

Figure 1 Screenshot of Genome Browser and SNP viewer displaying chromosome 13 of S. cerevisiae CEN.PK113-7D. The navigation panel (A) includes an overview of the genome, gene ID, gene name, gene function and the current location in the chromosome, together with exon and intron structure, and a text box for quick searching. Below this is a frameshowing the SNP viewer. (B) SNP information section for displaying nucleotide and amino acid polymorphism data between yeast $S$. cerevisiae strains.

A significantly higher number of metabolic SNPs, 939, were detected in CEN.PK113-7D and distributed across 158 unique metabolic genes, 85 of which contained a total 219 nonsynonymous SNPs.

In an effort to characterize the nonsynonymous metabolic SNPs identified in CEN.PK113-7D with biological significance, Gene Ontology (GO) process categorization was performed and presented in Figure 2 ranked according to statistical significance $(p<0.01)$. The most significant categories include carboxylic acid, organic acid, and carbohydrate metabolism, followed by nitrogen, amino acid, lipid, aromatic compound, and glycoprotein metabolism. Additional file 1 Figure S1 presents the GO function and component categorization, and as expected the highest significant concentration of nonsynonymous SNPs $(p<0.01)$ distributed across a specific enzyme class is for transferases. Notably, the background genes used for GO enrichment analysis were the complete gene set available in SGD (verified, unverified, and dubious). GO enrichment analysis uses a hypergeometric distribution with Multiple Hypothesis Correct to calculate $p$-values and correct for multiple sampling.

A graphical representation of all silent and nonsynonymous SNPs mapped to their specific metabolic pathways is presented in Additional file 1 Figure S2. Figure 3 highlights two metabolic pathways, galactose uptake and ergosterol synthesis, where an enrichment of nonsynonymous and silent SNPs was observed. Specifically, GAL1, GAL10, ERG8, and ERG9 contained nonsynonymous SNPs, while GAL7, ERG20 and HMG1 contained silent SNPs. The specific SNPs are identified as well the resulting amino acid substitutions.

In addition to identifying SNP enriched metabolic pathways in CEN.PK113-7D, an analysis intended to determine the prevalence of the SNP across the top 10 homologous sequences resulting from a multi-alignment 
Table 2 Genome sequencing and metabolic SNP detection

\begin{tabular}{|c|c|c|}
\hline Sequencing Parameter & S288c & CEN.PK113-7D \\
\hline \multicolumn{3}{|l|}{ MAQ Software Determination } \\
\hline No. of Reads & $5,301,907$ & $6,603,200$ \\
\hline Total No. of SNPs & 3,032 & 27,868 \\
\hline Total No. of Non-Ambiguous SNPs & 1,013 & 24,663 \\
\hline Total No. of Filtered SNPs ${ }^{A, B}$ & 311 & 13,787 \\
\hline \multicolumn{3}{|l|}{ Metabolism Focused Detection } \\
\hline Total No. of Metabolic Genes Considered ${ }^{c}$ & 782 & 782 \\
\hline Total No. of Metabolic Bases (bp) & $1.16 \mathrm{M}$ & $1.16 \mathrm{M}$ \\
\hline No. of Aligned Reads & 477,565 & 623,400 \\
\hline Total Gap Size (bp) & 0 & 0 \\
\hline Metabolic Genome Percent Reference Coverage (\%) & 99.7 & 99.4 \\
\hline Total No. of Metabolic SNPs detected & 36 & 939 \\
\hline Total No. Nonsynonymous Metabolic SNPs Detected & 3 & 219 \\
\hline Percent of SNPs Detected Nonsynonymous (\%) & 8.3 & 23.3 \\
\hline Total No. of Metabolic Genes Containing SNP & 14 & 158 \\
\hline Total No. of Metabolic Genes with Nonsynonymous SNP & 3 & 85 \\
\hline
\end{tabular}

Notes: Base pairs (bp). (A) Filtered SNPs determined based on cut-off criteria within the Mapping and Assembling with Quality (MAQ) software environment. (B) Detected SNPs with estimated error $<0.003 \%$ (C) The total number of genes classified as metabolic was based on the Saccharomyces Genome Database, Strain S288c, version 12.0. The "S288c" designation in Table 2 refers to the resequencing of S. cerevisiae S288c using Illumnia/Solexa sequencing technology.

Pfam query was performed. To better quantify those results, the parameters CEN.PK Match Frequency, Dominant AA Frequency, S288c Match Frequency, and Conservation Distance were defined and calculated (See Additional file 1 Figure S3). The Conservation Distance, bound between -1 and 1 , is a measure of whether the SNP identified in CEN.PK113-7D is more prevalent amongst homologous Pfam sequences (maximum Conservation Distance $=-1$ ), or if S288c (reference SGD sequence) is more prevalent (maximum Conservation Distance $=+1$ ). Additional file 1 Figure S4 presents the Conservation Distance across nonsynonymous SNPs identified, with the average value of $0.03 \pm 0.40(n=219)$, indicating that there is virtually no bias between S288c or CEN.PK113-7D as compared to their homologues. Extending this approach further, each amino acid polymorphism was characterized across a multi-alignment Pfam homologue search, and categorized according to standard amino acid properties (See Additional file 1 Figure S3). For example, Figure 4 presents SNPs identified in ERG8 at nucleotide positions 75 and 192. The resulting amino acid partially encoded by position 192 was $75 \%$ polar, $25 \%$ non-polar, $25 \%$ hydrophobic, and $75 \%$ hydroxylic looking across the top 10 Pfam homologous sequences. Lastly, and of most relevance to understanding the amino acid functional changes resulting from a SNP, the same categorization is presented for the S288c v. CEN.PK113-7D sequence. For example, the SNP at position 192 of ERG8 resulted in changing the encoded amino acid from non-polar (S288c) to polar (CEN. PK113-7D), and from hydrophobic (S288c) to hydroxylic
(CEN.PK113-7D). This approach is extended to all the ERG8 nonsynonymous SNPs as an example of extending nucleotide level changes to amino acid functional changes (See Additional file 1 Figure S5 for additional ERG8 nonsynonymous SNPs). Furthermore, Additional file 1 Figure S6 highlights functional changes for all metabolic nonsynonymous SNPs identified.

\section{Physiological Characterization}

The S. cerevisiae strains S288c and CEN.PK113-7D were physiologically characterized in both batch glucose and galactose supplemented fermentations. On glucose, CEN.PK113-7D exhibited a 32\% higher specific growth rate than S288c, correlating with the 33\% higher specific glucose consumption rate (See Table 3). The CEN. PK113-7D extracellular metabolic specific productivity rates were $32.6 \%$, 392\%, and $17.9 \%$ higher for ethanol, acetate, and glycerol production compared to S288c, respectively, while the specific oxygen consumption rates were nearly equivalent $\left(1.98 \mathrm{O}_{2}-\mathrm{mmol} \mathrm{g-DCW}{ }^{-1} \mathrm{~h}^{-1}\right.$ for CEN.PK113-7D v. 1.95 mmol-O $_{2}$ g-DCW ${ }^{-1} \mathrm{~h}^{-1}$ for S288c). Following complete glucose fermentation, as indicated by the peak carbon dioxide evolution rate (CER), both strains underwent a diauxic shift, clearly identified by the transition of the respiratory quotient (RQ) from $>1$ to $<1$, and ethanol accumulated during glucose fermentation (11.1 $\mathrm{g} \mathrm{L}^{-1}$ for CEN.PK113-7D v. $11.3 \mathrm{~g} \mathrm{~L}^{-1}$ for S288c) was respiro-fermented. The ethanol respiro-fermentation (ERF) phase (Figure 5) was clearly distinguishable in the CEN.PK113-7D compared to S288c, where both CER and oxygen uptake rates (OUR) 


\section{GO PROCESS, Nonsynonymous SNP Characterization}

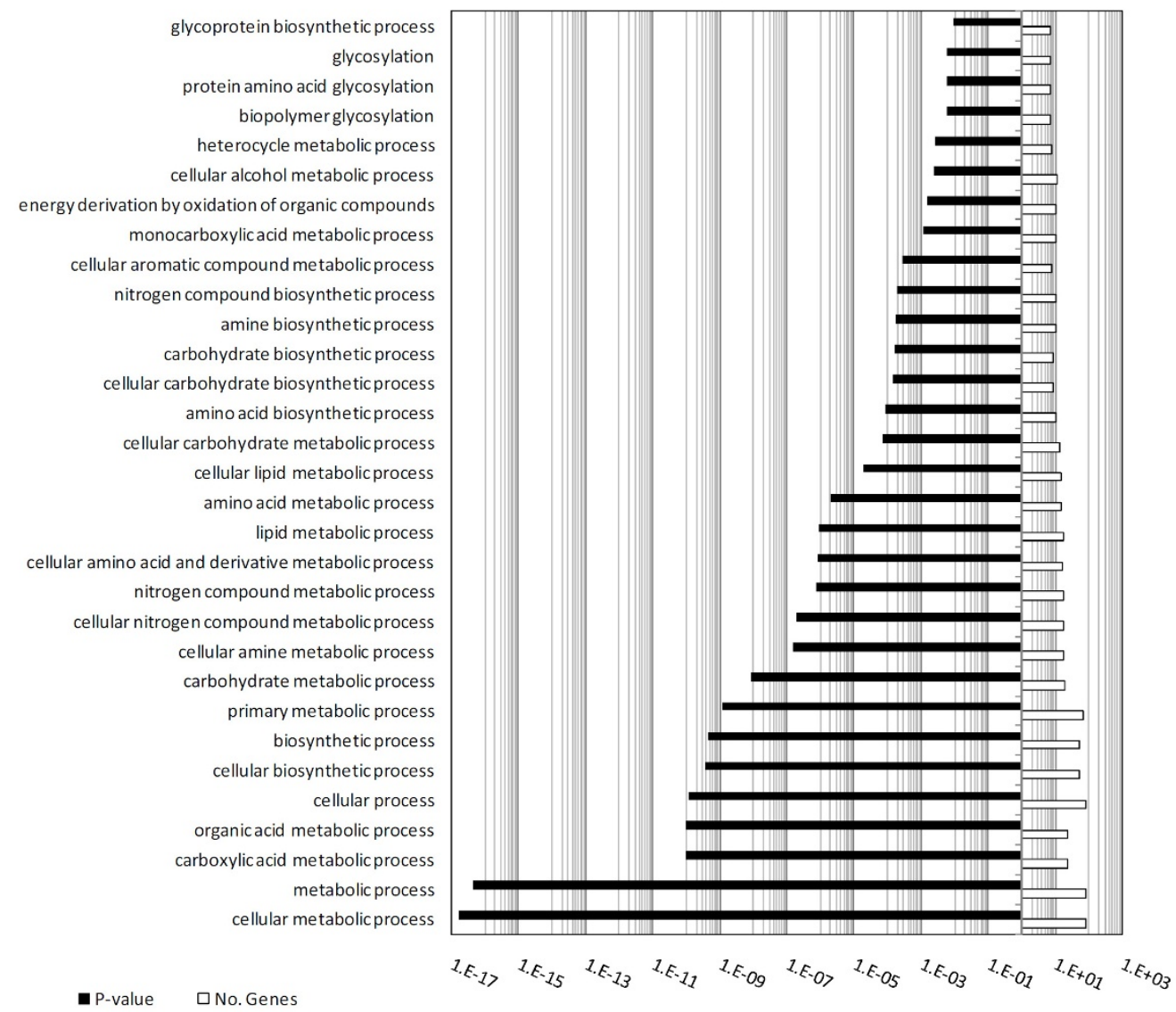

Figure 2 Gene Ontology (GO) process terms for the nonsynonymous SNPs identified in CEN.PK113-7D compared to S288c. The $x$-axis in log-scale displays both the significance of each category $(p<0.01$, symbol: solid black), and the number of genes from the total of 85 containing nonsynonymous SNPs (symbol: solid white). GO process characterization performed using the Saccharomyces Genome Database (SGD).

linearly increased, corresponding with the increase in biomass (3.7 to $12.0 \mathrm{~g}-\mathrm{DCW} \mathrm{L} \mathrm{L}^{-1}$ ). On the contrary, during the ERF phase for S288c there was a growth deficiency, clearly indicated by non-linear and significantly reduced CER and OUR rates, corresponding with a much lower increase in biomass $\left(2.1\right.$ to $6.9 \mathrm{~g}$-DCW L $\left.{ }^{-1}\right)$. The significantly decreased ERF phase in S288c compared to CEN.PK113-7D is also evident from the total time required to exhaust the ethanol ( $50 \mathrm{v} .33 \mathrm{~h}$, respectively).

A similar characterization was performed using batch galactose supplemented fermentations. CEN.PK113-7D demonstrated a slight lag-phase compared to glucose fermentation; however, sustained a galactose specific growth rate of $0.27 \mathrm{~h}^{-1}$ and galactose uptake rate of $24.3 \mathrm{C}$-mmol g-DCW ${ }^{-1} \mathrm{~h}^{-1}$, representing a $34 \%$ and $77 \%$ reduction, respectively, compared to glucose (See Table 3). All extracellular metabolic specific productivity rates were significantly decreased (ethanol, acetate, and glycerol were $93 \%, 6.8 \%$, and $88 \%$ reduced compared to glucose, respectively), with the exception of OUR, which was $47 \%$ higher on galactose compared to glucose, leading to an effectively lower RQ of 1.5 compared to 11.9 during glucose cultivation. Furthermore, given the significantly lower RQ during the exponential phase of galactose fermentation, relatively little ethanol was produced $\left(2.7 \mathrm{~g} \mathrm{~L}^{-1}\right)$, resulting in a short ERF phase ( $<5$ h) (See Figure 5). Similarly, S288c was cultivated on galactose; however, a significant deficiency in the strain's ability to metabolize this carbon source was observed. A total of $25 \mathrm{~h}$ post-inoculation elapsed with no increase in biomass as compared to CEN.PK113-7D where after $6 \mathrm{~h}$ post-inoculation two cell doublings were observed. At $25 \mathrm{~h}$ post-inoculation a glucose bolus of $10 \mathrm{~g} \mathrm{~L}^{-1}$ was added to promote growth, and rapidly, glucose fermentation, a diauxic shift, and ethanol respiro-fermentation were 


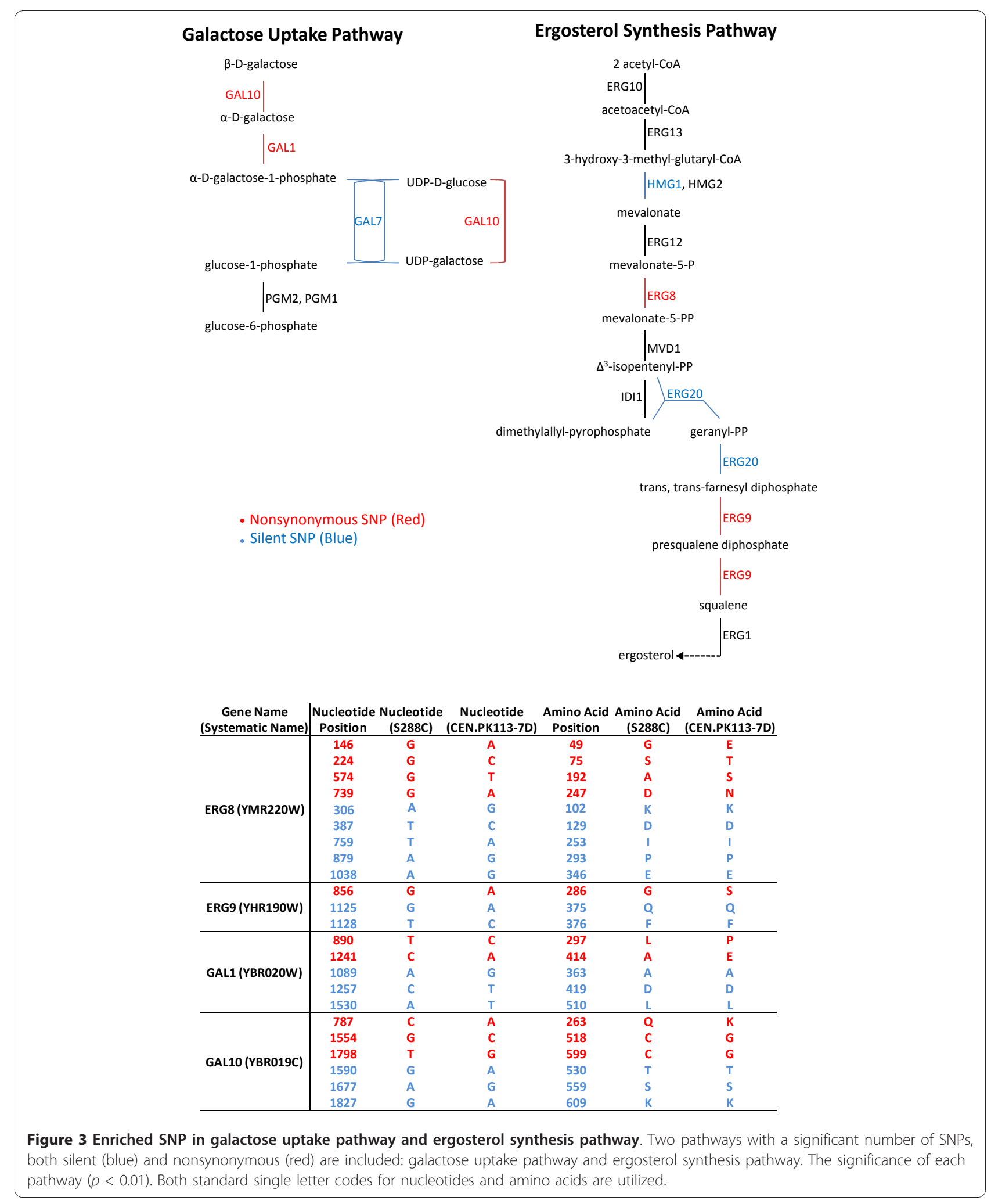



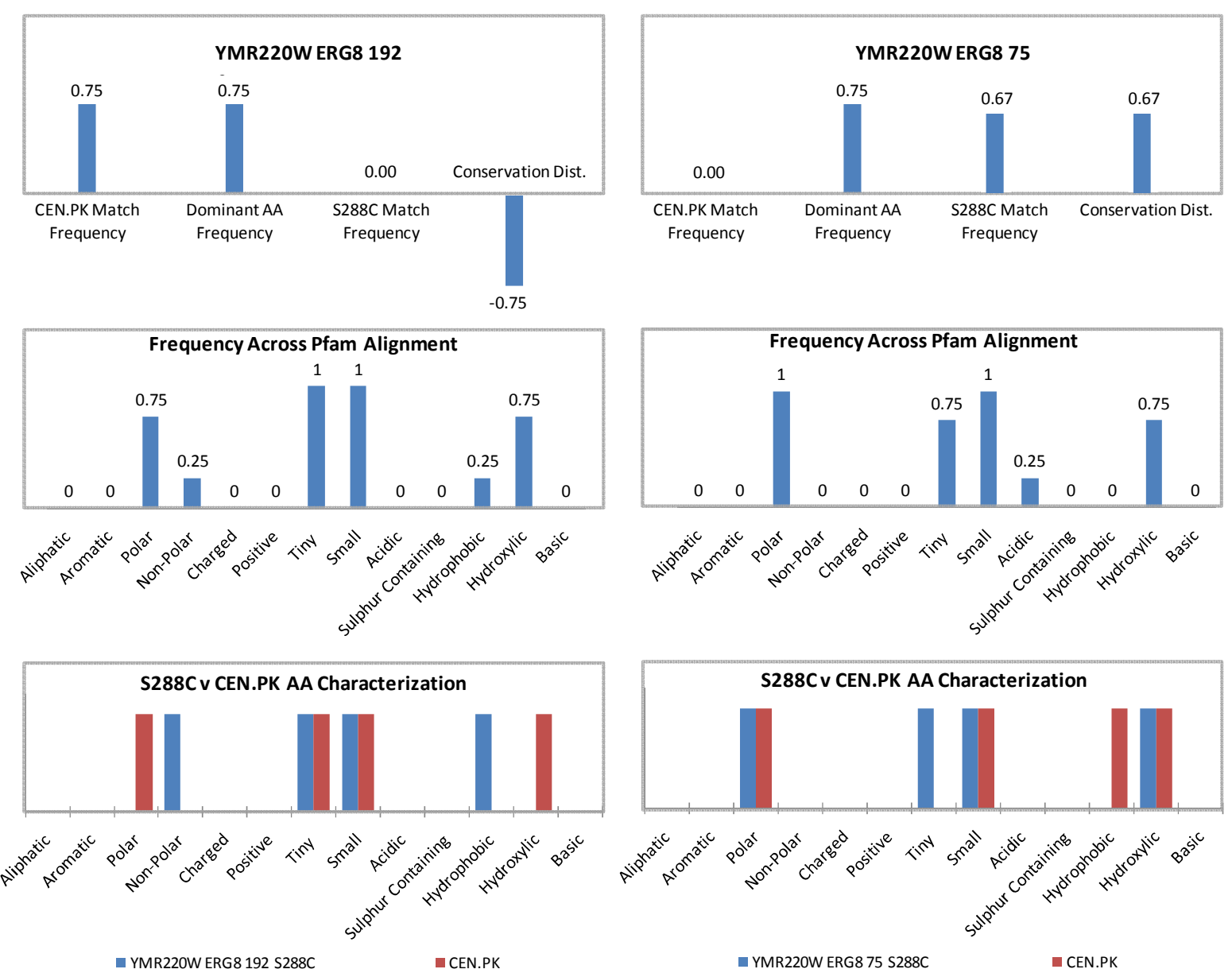

Figure 4 An example of amino acid properties change of CEN. PK113-7D compared to S288c: the gene ERG8 of the ergosterol synthesis pathway. The gene ERG8 of the ergosterol synthesis pathway contains a total of 4 nonsynonymous SNPs, two of which, located at nucleotide positions 192 and 75, are analyzed here. The top plots show the CEN.PK Match Frequency, Dominanat AA Frequency, S288c Match Frequency, and Conversation Distance. The middle plots show the frequency (fraction) of each categorization across the amino acid sequences resulting from Pfam multi-sequence alignment. The bottom plots shows the characterization of the original S288c amino acid (symbol: blue bar) and the CEN.PK113-7D amino acid (symbol: red bar). The gene ERG8 contained a total of 4 nonsynonymous SNPs, and Additional file 1, Figure S5 includes the other 2 nonsynonymous SNPs (nucleotide positions 49 and 247).

observed (See Figure 5). Both co-consumption of galactose and ethanol, and a galactose only respiro-fermentative (GaRF) growth phase were observed. During co-consumption the specific growth rate was $0.14 \mathrm{~h}^{-1}$, while on galactose only the specific growth rate was $0.02 \mathrm{~h}^{-1}$. Similarly, the extracellular specific metabolite productivity rates were nearly zero when only galactose consumption was considered (See Table 3). Ethanol was consumed by $82 \mathrm{~h}$ postinoculation, and in the period from $82 \mathrm{~h}$ to $128 \mathrm{~h}$, only galactose consumption was observed, and biomass increased from $7.9 \mathrm{~g}$-DCWL L ${ }^{-1}$ to $20.9 \mathrm{~g}$-DCW L $\mathrm{L}^{-1}$, representing a doubling time of $35 \mathrm{~h}$ compared to $2.6 \mathrm{~h}$ for CEN. PK113-7D.

For each cultivation condition and strain, ergosterol measurements were performed and presented in Figure 6. At the same time of transcriptome sampling, which occurred during mid-exponential phase of glucose fermentation (18-

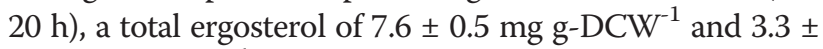
$0.5{\mathrm{mg} \mathrm{g}-D C W^{-1}}$ for CEN.PK113-7D and S288c, respectively, was measured. Subsequently, the diauxic shift and ERF phase was characterized by two ergosterol samples during early and mid-ERF phase, and followed by a final (stationary) sample post-ethanol exhaustion. S288c ergosterol content was significantly higher during ethanol metabolism as compared to CEN.PK113-7D, but post-ethanol metabolism CEN.PK113-7D exhibited a significantly higher ergosterol content $\left(15.9 \pm 0.7 \mathrm{mg}\right.$ g-DCW ${ }^{-1}$ v. $2.6 \pm 0.07$

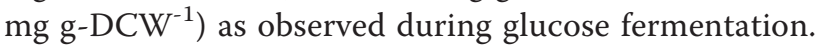
For galactose cultivations, ergosterol content was only measured during transcriptome sampling, which occurred 
Table 3 Physiological characterization of S. cerevisiae strains S288C and CEN.PK113-7D

\begin{tabular}{|c|c|c|c|c|c|c|c|c|c|c|}
\hline \multirow{3}{*}{$\begin{array}{l}\text { Strain } \\
\text { Substrate }\end{array}$} & \multicolumn{2}{|c|}{$\mathrm{S} 288 \mathrm{c}$} & \multicolumn{2}{|c|}{$\begin{array}{l}\text { CEN.PK } \\
\text { 113-7D }\end{array}$} & \multicolumn{2}{|c|}{ S288c } & \multicolumn{2}{|c|}{$\mathrm{S} 288 \mathrm{c}$} & \multicolumn{2}{|c|}{$\begin{array}{l}\text { CEN.PK } \\
113-7 D\end{array}$} \\
\hline & \multicolumn{2}{|c|}{ Glucose } & \multicolumn{2}{|c|}{ Glucose } & \multicolumn{2}{|c|}{ Galactose } & \multicolumn{2}{|c|}{$\begin{array}{l}\text { Galactose/ } \\
\text { Ethanol }\end{array}$} & \multicolumn{2}{|c|}{ Galactose } \\
\hline & Mean & $\begin{array}{r} \pm S D \\
(n=2)\end{array}$ & Mean & $\begin{array}{r} \pm S D \\
(n=2)\end{array}$ & Mean & $\begin{array}{r} \pm S D \\
(n=2)\end{array}$ & Mean & $\begin{array}{r} \pm S D \\
(n=2)\end{array}$ & Mean & $\begin{array}{c} \pm S D \\
(n=2)\end{array}$ \\
\hline$\mu-\max \left(h^{-1}\right)$ & 0.31 & 0.01 & 0.41 & 0.01 & 0.02 & 0.00 & 0.14 & 0.01 & 0.27 & 0.00 \\
\hline Carbon Recovery (\%) & 96.60 & 1.90 & 95.50 & 3.90 & $\mathrm{n} / \mathrm{a}$ & $\mathrm{n} / \mathrm{a}$ & $\mathrm{n} / \mathrm{a}$ & $\mathrm{n} / \mathrm{a}$ & 79.60 & 2.60 \\
\hline \multicolumn{11}{|c|}{ Specific Productivity or Consumption Rate ${ }^{A}$} \\
\hline$-r_{\text {Gluc }}$ or $-r_{\text {Gal }}$ & 79.35 & 5.48 & 105.15 & 0.24 & 1.21 & 0.57 & 4.50 & 0.70 & 24.28 & 0.33 \\
\hline $\mathrm{r}_{\mathrm{CO} 2}$ & 18.36 & 0.52 & 23.62 & 0.87 & 0.11 & 0.01 & 0.58 & 0.12 & 4.31 & 0.24 \\
\hline $\mathrm{r}_{\mathrm{EtOH}}$ & 37.59 & 4.39 & 49.88 & 0.34 & 0.01 & 0.01 & -3.97 & 0.62 & 3.50 & 0.26 \\
\hline$r_{\text {Acet }}$ & 0.24 & 0.02 & 1.18 & 0.04 & 0.00 & 0.00 & -0.04 & 0.02 & 1.10 & 0.11 \\
\hline$r_{\text {Glyc }}$ & 6.08 & 0.94 & 7.17 & 2.64 & 0.00 & 0.00 & -0.58 & 0.05 & 0.89 & 0.09 \\
\hline$r_{P y r}$ & 0.47 & 0.04 & 0.69 & 0.06 & 0.00 & 0.00 & 0.00 & 0.00 & 0.04 & 0.01 \\
\hline$r_{\text {Suc }}$ & 0.01 & 0.01 & 0.03 & 0.05 & 0.00 & 0.00 & 0.00 & 0.00 & 0.00 & 0.00 \\
\hline$r_{x}$ & 13.85 & 1.11 & 17.79 & 0.02 & 0.78 & 0.05 & 5.85 & 0.02 & 9.49 & 0.89 \\
\hline$-\mathrm{r}_{\mathrm{O} 2}$ & 1.95 & 0.07 & 1.98 & 2.75 & 0.08 & 0.01 & 0.97 & 0.02 & 2.91 & 0.22 \\
\hline
\end{tabular}

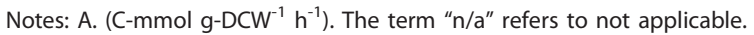

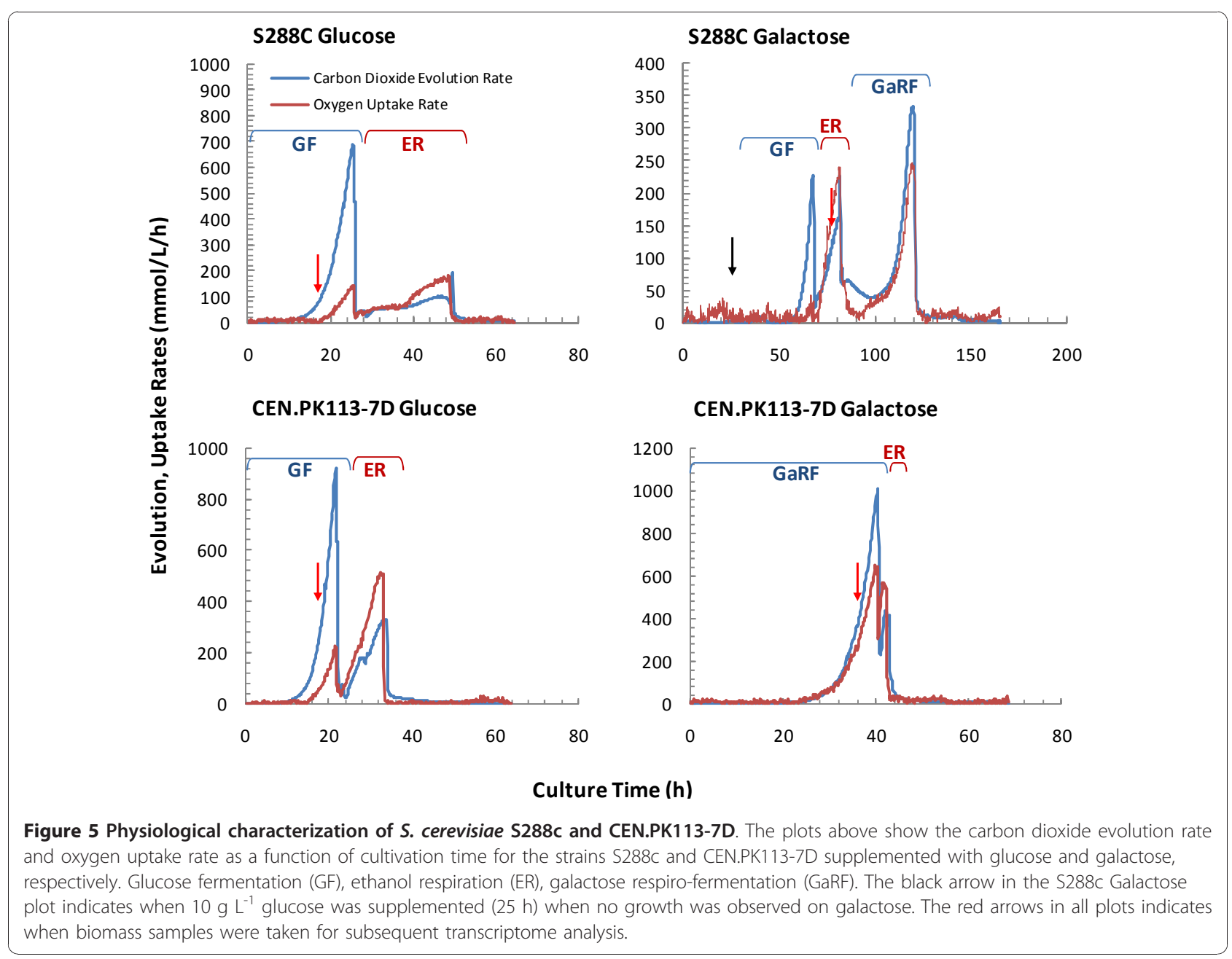



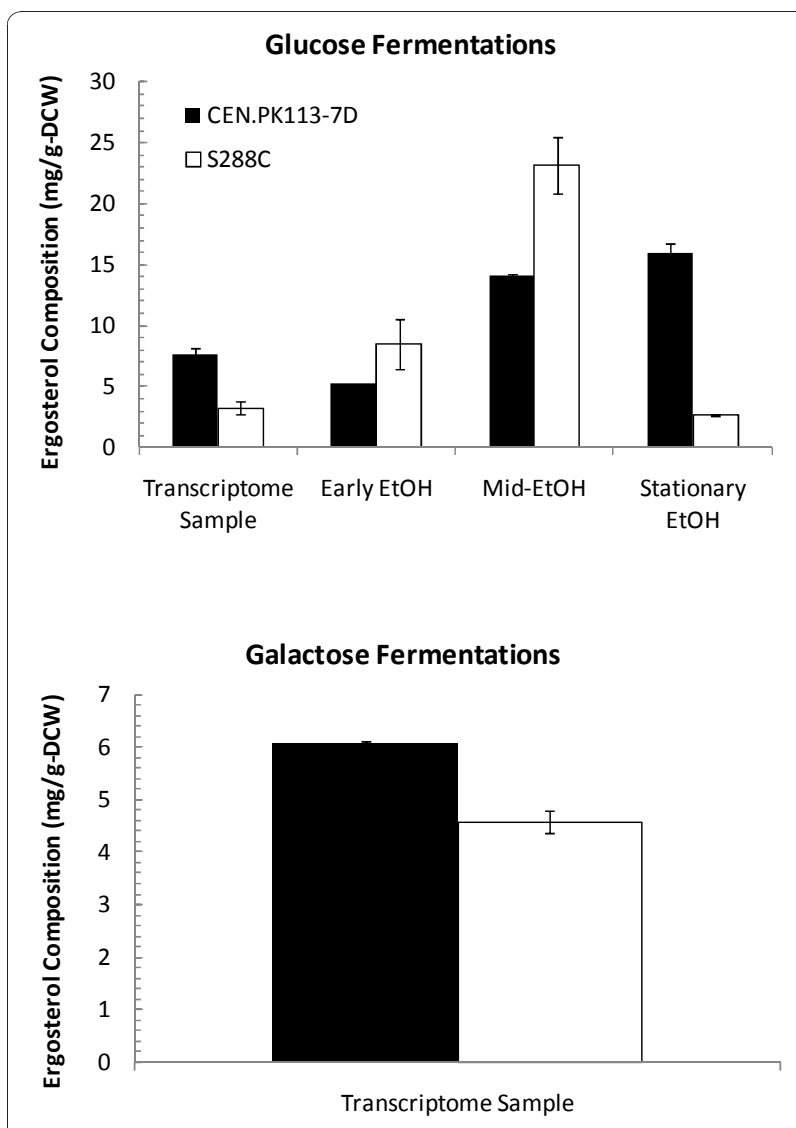

Figure 6 Ergosterol measurements in S. cerevisiae strains S288c and CEN.PK113-7D. Ergosterol composition (mg g-DCW ${ }^{-1}$ ) was measured for different samples taken during S288C and CEN.PK1137D fermentations, supplemented with glucose and galactose. Transcriptome sample was taken during the mid-exponential fermentation phase on glucose or respiration phase on galactose. For glucose fermentations, early ethanol, mid-ethanol, and stationary ethanol samples were taken post-diauxic shift to charcterize the change in ergosterol during growth on ethanol. Error bars are \pm SD $(n=2)$.

at $78 \mathrm{~h}$ for S288c (co-consumption of ethanol and galactose observed), and $35 \mathrm{~h}$ for CEN.PK113-7D. The total

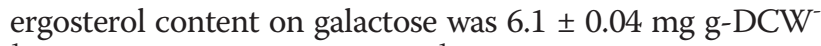
1 and $4.6 \pm 0.2 \mathrm{mg} \mathrm{g}-\mathrm{DCW}^{-1}$ for CEN.PK113-7D and $\mathrm{S} 288$ c, respectively.

\section{Transcriptome Characterization}

Differential gene expression between S288c and CEN. PK113-7D, cultivated on both glucose and galactose, is summarized in Table 4. The GO characterization (process, function, component) for the comparative conditions S288c v. CEN.PK113-7D cultivated on glucose, and S288c v. CEN.PK113-7D cultivated on galactose, and divided into $\log _{2}$-fold change (lfc) $>0$ and $<0$, is presented in Additional file 1 Figure S7 and Figure S8. The metabolic pathway expression maps for each comparative condition are included in Additional file 1 Figure S9 and Figure S10. Lastly, all genes exhibiting statistically significant differential gene expression $\left(p_{a d j}<0.01\right)$ and having either a silent or nonsynonymous SNP are included in Additional file 1 Table S1 and Table S2.

For the condition S288c v. CEN.PK113-7D cultivated on glucose, the top 272 differentially expressed genes, ranked according to $p_{a d j}$ value are characterized into GO process terms largely dominated by responses to stimuli and pheromone, with the dominant metabolic process categories being trehalose metabolism, steroid metabolism, and amino acid transport. Specific genes consistent with this categorization high in $p_{a d j}$ value rank and lfc $>0$ are GSY1 (glycogen synthase, lfc 2.0, $p_{a d j}$ value rank 23) and for lfc < 0 is HMG1 (HMG-CoA reductase, lfc $-1.7, p_{a d j}$ value rank 14). For the condition S288c v. CEN.PK113-7D cultivated on galactose, the top 501 differentially expressed genes, ranked according to $p_{a d j}$ value are characterized into GO process terms response to simuli and stress, carbohydrate metabolism, and transport. Specific metabolic genes noteworthy in this category, high in $p_{a d j}$ value rank amongst genes with lfc $>0$ include $M D H 2$ (malate dehydrogenase, lfc 2.8, $p_{\text {adj }}$ value rank 8), FBP1 (fructose-1,6-bisphosphatase, lfc $4.2, p_{a d j}$ value rank 15), GAD1 (glutamate decarboxylase, lfc 3.0, $p_{\text {adj }}$ value rank 30), GDH3(NADP ${ }^{+}$ dependent glutamate dehydrogenase, lfc 3.2, $p_{a d j}$ value rank 32), GSY1 (lfc 1.4, $p_{a d j}$ value rank 41), and ICL1 (isocitrate lyase, lfc 2.7, $p_{\text {adj }}$ value rank 54). Similarly, specific metabolic genes high in $p_{a d j}$ value rank amongst genes with lfc $<0$ include $A R E 2$ (acyl-coA:sterol acetyltransferase, lfc -2.3, $p_{\text {adj }}$ value rank 10), and CYB5 (cytochrome b5, lfc -1.6, $p_{\text {adj }}$ value rank 47).

In an effort to further investigate if larger regulatory mechanisms could be identified the list of genes exhibiting significant differential expression were submitted to the Yeast Search for Transcriptional Regulators And Consensus Tracking (YEASTRACT) curated repository of associations between transcription factors and target genes in $S$. cerevisiae $[25,26]$. The transcription factor, Tec1p, was identified as directly regulating $21.1 \%$ of the total submitted gene list (See Table 4, 272 genes, S288c glucose v. CEN.PK113-7D glucose), and was 1.7-fold higher expressed in CEN.PK113-7D compared to S288c ( $p_{\text {adj }}$ value $=7.2 \times 10^{-3}$ ). Tec1p was the only identified transcription factor to be significantly differentially expressed, and strongly regulates FLO11, a flocculin gene required for invasive growth, and pseudohyphal formation [27]. The transcription factors regulating the highest percentage of the differentially expressed genes, yet not being differentially expressed themselves, were Sok $2 \mathrm{p}$ and Ste12p, with $32.5 \%$ and $21.5 \%$, respectively, of submitted genes being directly regulated. Sok2p and Ste12p are transcription factors negatively regulating pseudohyphal differentiation 
Table 4 Summary of Differential Gene Expression

\begin{tabular}{|c|c|c|}
\hline \multirow[t]{2}{*}{ Summary of Differential Expression $\left(p_{a d j}<0.01\right)$} & \multicolumn{2}{|c|}{ S288c v. CEN.PK113-7D } \\
\hline & Glucose $(\mathrm{n}=2)$ & Galactose $(\mathrm{n}=2)$ \\
\hline Total No. of Differentially Expressed Genes & 272 & 501 \\
\hline No. of Genes LFC > 0 & 204 & 337 \\
\hline $\mathrm{LFC} \pm \mathrm{SD}$ & $2.13 \pm 1.41$ & $1.81 \pm 1.17$ \\
\hline No. of Genes with SNPs Detected & 13 & 17 \\
\hline No. of Genes with Nonsynonymous SNPs & 7 & 10 \\
\hline \multicolumn{3}{|l|}{ No. of Genes $L F C<0$} \\
\hline $\mathrm{LFC} \pm \mathrm{SD}$ & $-2.12 \pm 1.32$ & $-1.53 \pm 1.05$ \\
\hline No. of Genes with SNPs Detected & 4 & 4 \\
\hline No. of Genes with Nonsynonymous SNPs & 1 & 0 \\
\hline
\end{tabular}

Notes: Single nucleotide polymorphism (SNP). Standard deviation (SD). Nonsynonymous SNPs defined as a nucleotide modification results in a translated amino acid modification.

[10]. A similar analysis was performed for galactose; however, similar results were obtained, with Sok $2 p$ and Ste12p directly regulating $23.1 \%$ and $17.4 \%$, respectively, of the 501 differentially expressed genes (See Table 4). The transcription factors differentially expressed themselves were Msa1p $\left(p_{a d j}\right.$ value $\left.=1.37 \times 10^{-1}\right)$ and Msa2p $\left(p_{\text {adj }}\right.$ value $=$ $\left.1.65 \times 10^{-1}\right)$, putative G1-specific cell cycle transcription activators, and Usv1p, a putative zinc finger transcription factor regulating growth on non-fermentable carbon sources. USV1 expression was 2.2-fold higher in CEN. PK113-7D compared to S288c $\left(p_{\text {adj }}\right.$ value $\left.=3.6 \times 10^{-3}\right)$. Although relatively little is known about Usv1p, it has been shown to be induced post-diauxic shift, consistent with the deficiency in post-diauxic shift metabolism observed in S288c [28]. With the exception of Usv1p, all transcription factors identified are more closely related to the significant difference in growth rates between strains rather than their respiro-fermentative metabolism. Metabolic SNPs identified and subsequent analysis did not identify clear correlations or pathway enrichment that could explain the lack of respiro-fermentative metabolism in S288c. Metabolic genes containing nonsynonymous SNPs in CEN.PK113-7D, significantly differentially expressed on galactose, and related to oxidative metabolism included ACS1 (Acetyl-CoA synthetase), GAD1 (Glutamate decarboxylase), YAT2 (Carnitine acetyltransferase), and CCP1 (Mitochondrial cytochrome-c peroxidase) which were 7.8-fold, 3.2-fold, 4.5-fold, and 2.1-fold higher in CEN.PK113-7D, respectively (See Additional file 1 Table S2).

\section{Discussion}

The physiological characterization clearly suggests that S288c has a deficiency in metabolism of respiro-fermentative carbon sources, such as ethanol and galactose, when compared to CEN.PK113-7D. Inspection of the significantly differentially expressed genes between strains cultivated on glucose or galactose did not reveal an obvious gene cluster that would explain this significant physiological difference. This is supported both by the GO characterization and pathway expression mapping.

There were two central carbon metabolic pathways enriched with nonsynonymous SNPs that also correlated with significant differences in phenotype. S. cerevisiae CEN.PK113-7D exhibited significantly higher ergosterol content during growth on glucose, and to a lesser extent, galactose. This is consistent with previous work where CEN.PK2-1C had very high ergosterol/erg-ester $(20.0 \mathrm{mg}$ $\left.\mathrm{g}-\mathrm{DCW}^{-1}\right)$ and triacylglycerols content $\left(15.2 \mathrm{mg} \mathrm{g-DCW}^{-1}\right)$ compared to 9 other S. cerevisiae strains, including FY169 (ergosterol/erg-ester content: $8.5 \mathrm{mg} \mathrm{g-DCW}^{-1}$; triacylglycerols content: $2.4 \mathrm{mg}$ g-DCW ${ }^{-1}$ ) which is isogenic to S288c $[29,30]$. The ergosterol biosynthetic pathway had significant nonsynonymous SNPs identified in ERG8 and ERG9, and silent SNPs identified in ERG20 and HMG1. Both ERG8 and ERG9 were not significantly differentially expressed, either in glucose or galactose, suggestive again that phenotypic observations, consistent with genome sequence variations, are not necessarily directly manifested at the transcriptome level. Both ERG8 (encodes phosophomevalonate kinase) and ERG9 (encodes squalene synthesase) are essential cytosolic enzymes in the biosynthetic pathway of isoprenoids and sterols ( $\Delta \operatorname{erg} 8$ and $\Delta \operatorname{erg} 9$, were found to both be auxtrophic for ergosterol in the systematic deletion library), including ergosterol, from mevalonate $[11,31,32]$. The ergosterol biosynthetic pathway is highly regulated through feedback inhibition mechanisms and by several rate-controlling steps, including that catalyzed by HMG-CoA reductase, encoded by HMG1 [33,34]. Under both glucose and galactose, HMG1 expression was significantly down-regulated in S288c compared to CEN.PK113- 
7D by 3.2-fold $\left(p_{\text {adj }}\right.$ value $\left.=3.3 \times 10^{-4}\right)$ and 1.8 -fold $\left(p_{\text {adj }}\right.$ value $\left.=8.6 \times 10^{-3}\right)$, respectively, correlating with the significantly less ergosterol content in S288c cultivated on glucose and to a lesser extent, on galactose. Furthermore, $E R G 9$ has been previously identified as also having a regulatory role [35], consistent with the hypothesis that a nonsynonymous SNP resulting in altered protein function could affect ergosterol synthesis. ERG8 on the other hand has not been explicitly shown to have a regulatory function, yet, when the specific activity of $0.06 \mu \mathrm{mol} \mathrm{min}^{-1} \mathrm{mg}^{-}$

${ }^{1}$ is compared to other ergosterol synthetic enzymes such as ERG13 (2.1 in S. cerevisiae), ERG12 (0.77 in S. cerevisiae), ERG20 (5.22 in S. cerevisiae), and especially the known regulator HMG1/HMG2 (0.0035 in S. cerevisiae), it is suggestive that ERG8 is likely a rate limiting step [36-44]. There were a large number of nonsynonymous SNPs that encoded significant changes in amino acid classes, further suggestive that ERG8 is a strong metabolic engineering target for understanding the significantly higher ergosterol content in CEN.PK113-7D. Lastly, the observation that neither ERG8 nor ERG9 were differentially expressed under glucose or galactose, suggests their potential affect on phenotype is likely post-translational.

Similar to ergosterol biosynthesis, the galactose uptake pathway phenotype in S288c was vastly lower rate compared to CEN.PK113-7D, correlating with the nonsynonymous SNP enrichment in GAL1 and GAL10, and silent SNPs in GAL7. Neither GAL1 (encodes galactokinase) nor GAL10 (encodes UDP-glucose-4-epimerase) were significantly differentially expressed during growth on galactose; however, on glucose GAL1 was significantly up-regulated $\left(p_{\text {adj }}\right.$ value $\left.=9.7 \times 10^{-4}\right)$, 2.9-fold in CEN.PK113-7D. Both $\Delta$ gal1 and $\Delta$ gal10 mutants are unable to grow on galactose as sole carbon sources [45-47]. The significant number of nonsynonymous SNPs in both essential galactose genes suggests obvious targets for explanation of why S288c is incapable of galactose respiro-fermentative metabolism. Furthermore, it should be noted that S288c has been described as $\Delta$ gal2 (See Additional file 1 Table S3), which may be ascribed to the presence of 4 SNPs between CEN. PK113-7D and S288c in the coding region, and 14 SNPs in the upstream region that result in a poor functional Gal2p. However, clearly, S288c is able to cometabolize galactose with ethanol and this may be ascribed by galactose transport by e.g. the hexose transporters.

A further metabolic engineering benefit of whole genome sequencing was the detection of a nonsynonymous SNP resulting in a stop codon of PAD1 (encodes phenylacrylic acid decarboxylase). Pad1p is essential for decarboxylation of aromatic carboxylic acids conferring resistance to cinnamic acid, and a nonsynonymous SNP was detected at nucleotide position 294 ( $\mathrm{T}$ to $\mathrm{G}$ ), resulting in a stop codon (TAT $\rightarrow$ TAG) [48]. Although Pad1 relevant phenotypes were not explored, the transcriptome response on glucose revealed significant differential expression of PAD1 $\left(p_{\text {adj }}\right.$ value $\left.=1.5 \times 10^{-3}\right)$, with 3.1 -fold higher expression in S288c compared to CEN.PK113-7D. This is consistent with the stop codon detected in CEN. PK113-7D at position 294, noting that the total ORF genomic DNA sequence is 729 nucleotides, and therefore unlikely to be transcribed and detected.

\section{Conclusions}

Whole high-throughput genome sequencing of S. cerevisiae S288c and CEN.PK113-7D resulted in identification of 13,787 filtered SNPs in CEN.PK113-7D, with a total of 939 SNPs detected across 158 unique metabolic genes, 85 of which contained a total of 219 nonsynonymous SNPs. There were two central carbon metabolic pathways enriched with nonsynonymous SNPs that also correlated with significant differences in phenotype. $S$. cerevisiae CEN.PK113-7D exhibited significantly higher ergosterol content during growth on glucose, and to a lesser extent, galactose. The ergosterol biosynthetic pathway had significant nonsynonymous SNPs identified in ERG8 and ERG9, and silent SNPs identified in ERG20 and HMG1. The flux through the galactose uptake pathway was much lower in S288c compared with CEN. PK113-7D, correlating with the nonsynonymous SNP enrichment in GAL1 and GAL10, and silent SNPs in GAL7. More globally, the physiological characterization clearly suggests that S288c has a deficiency in metabolism of respiratory carbon sources, such as ethanol and galactose, when compared to CEN.PK113-7D. Inspection of the significantly differentially expressed genes between strains cultivated on glucose or galactose did not reveal an obvious gene cluster that would explain this significant physiological difference. In summary and perhaps not surprisingly, transcriptome analysis did not provide a clear hypothesis for major phenotypes observed, suggesting that genotype to phenotype correlations are manifested post-transcriptionally or posttranslationally either through protein concentration and/ or function. Clearly, future work must validate these correlations through genetic engineering of identified SNPs in either S288c or CEN.PK113-7D to see if desired phenotypes, such as increased galactose uptake or ergosterol synthesis in S288c, are observed. Future work must also expand on the metabolic SNP analysis presented to include all 13,787 SNPs, realizing phenotypic observations may not necessarily be linked directly to metabolic SNPs, but rather SNPs affecting larger regulatory mechanisms and networks, such as those governed by transcription factors. Certainly, as $S$. cerevisiae continues to be exploited, particularly for metabolic engineering 
applications, the integration of physiological characterization, transcriptome analysis, and metabolic SNP detection with high-throughput whole genome sequencing provides direct correlations between observed phenotypes and genotypes and offers high probability of success metabolic targets.

\section{Methods}

\section{Strain Description}

S. cerevisiae strain S288c (American Type Culture Collection, $\mathrm{ATCC}^{\odot}$ ) and strain CEN.PK113-7D (Scientific Research and Development, SRD GmbH were used in this study. Genotype of strain S288c was described by Mortimer et al, [49], Johnston et al [50], Goffeau et al [10], Cherry et al [11] and genotype of strain CEN. PK113-7D was described by Cherry et al [11], van Dijken et al [24]. More information of S. cerevisiae strains was described in Additional file 1 Table S3.

\section{Genome Sequencing, Annotation and Analysis DNA Isolation}

A standard $500 \mathrm{~mL}$ shake flask, supplemented with $10 \mathrm{~g}$ $\mathrm{L}^{-1}$ glucose and inoculated with a single colony of $S$. cerevisiae S288c or CEN.PK113-7D, was permitted to grow for $24-48 \mathrm{~h}$ at $30^{\circ} \mathrm{C}$ until visual inspection confirmed a high optical density. A total of $5 \mathrm{~mL}$ culture was aliquoted into $15 \mathrm{~mL}$ sterile tubes (one per extraction), centrifuged (4000 RCF) for $5 \mathrm{~min}$, washed with $2 \mathrm{~mL}$ deionized water, and pelleted. Cell pellets were resuspended in $0.5 \mathrm{~mL}$ lysis buffer. Lysis buffer consisted of $0.1 \mathrm{M}$ Tris $\mathrm{pH}$ 8.0, $50 \mathrm{mM}$ EDTA, and 1\% SDS final concentration. The lysis buffer suspension was transferred to a $1.5 \mathrm{~mL}$ FastPrep screw cap tube, to which $200 \mu \mathrm{L}$ acid-washed glass beads (250-500 $\mu \mathrm{m})$ and 25 $\mu \mathrm{L} 5 \mathrm{M} \mathrm{NaCl}$ was added. A FastPrep ${ }^{\mathrm{TM}}$ FP120 (QBiogene, Irwine, CA) was used for cell lysis, with two cycles of 20 $\mathrm{s}$ disruption and $1 \mathrm{~min}$ on ice. The resulting cell suspension was centrifuged (13,000 RCF) for $10 \mathrm{~min}$., and the resulting clear liquid, approximately $350 \mu \mathrm{L}$, avoiding white cell debris and beads, was aspirated with a pipette and transferred to $1.5 \mathrm{~mL}$ microcentrifuge tubes. $400 \mu \mathrm{L}$ chloroform (TE-saturated) was added to each tube, mixed, and a chloroform extraction performed. $1 \mathrm{~mL}$ 99\% ethanol was added to the resulting suspension, mixed, centrifuged (13,000 RCF) for 6 min., ethanol decanted, and then resuspended in $70 \%$ ethanol. The resulting suspension was centrifuged (13,000 RCF) for 6 min., ethanol decanted, and pellet permitted to dry for 25-60 min. The pellet was then resuspended in $50 \mu \mathrm{L} 2 \mathrm{mM}$ Tris, incubated for $10 \mathrm{~min}$. at $37^{\circ} \mathrm{C}$, and stored at $-20^{\circ} \mathrm{C}$. Illumina/Solexa Genome Sequencing and SNP Analysis Isolated DNA from S. cerevisiae S288c and CEN.PK1137D were processed to prepare short insert shotgun libraries at Fasteris SA (Geneva, Switzerland), utilizing the Solexa technology according to the manufacturer's recommendations (Illumina). The whole-genome sequencing was performed in 2007 on a Genome Analyzer "classic" instrument with sequencing kits version 1 and base calling on the Solexa Pipeline (version 0.2.2.5). SNP detection was performed using two independent approaches: mapping on the S288c reference sequence and de novo assembly. The MAQ software package http://maq.sourceforge.net was used to map the short base paired-reads (35 bp) to the reference genome S. cerevisiae S288c version 12.0, available at the Saccharomyces Genome Database (SGD). For consensus calling, the $m a q$ assemble $-m$ command was used to call the consensus sequences from read mapping. The value $m$ set to 1 specifies the maximum numbers of mismatches allowed for a filtered read. To detect high-quality SNP identification, the command maq.pl SNPfilter was set up. The threshold values applied to SNP detection for the CEN.PK113-7D sequence relative to the reference sequence were read depth $(-d)>5$ for few reads, read depth $(-D)<255$ for too many reads, uniqueness of the region $(-w)<1.5$, quality of the read $(-Q)>50$, and best read quality $(-n)>40$. These threshold parameter values were tested such that the amount of coverage and proportion of genome with aligned sequences was maximized, and a graphical representation of SNPs was produced to confirm results. The de novo assembly has been performed using the EDENA software package http://www.genomic.ch/edena.php as described previously [21]. The EDENA assembly results for both whole genome sequencing and for SNP detection in metabolic genes are presented in Additional file 1 , Table S4. The de novo assembly used for SNP detection was essentially used for an independent verification of the SNPs detected by mapping on the reference sequence, as not all the genome was represented. Indeed a higher coverage, as well as longer insert libraries are required to achieve more complete de novo assembly. For purposes of the subsequent SNP analysis only the MAQ software results were used. The FASTA files of each genome sequence are available upon request.

All metabolic genes containing SNPs, both silent and nonsynonymous, were manipulated within the software BioEdit v7.08 http://www.mbio.ncsu.edu/BioEdit/bioedit. html. Specifically, the ORF genomics nucleotide sequence available on Saccharomyces Genome Database (SGD) http://www.yeastgenome.org were imported into BioEdit, and the sequences modified with the identified SNP, creating a new CEN.PK113-7D sequence for that ORF relative to the original S288c strain. Both the S288c and CEN.PK113-7D nucleotide sequences were then translated in fix full frames, and amino acid polymorphisms were identified, leading to the categorization of each SNP as either being silent or nonsynonymous. 
Subsequent physiological characterization of the gene and all relevant amino acid information from UnitProt were managed in a spreadsheet using Microsoft Excel. Multi-sequence Pfam alignments were performed using a custom BioPerl script and the UNIX operating environment. Calculations and characterization described in Additional file 1, Figure S3, related to amino acids, were then performed using Microsoft Excel.

\section{Gene Prediction and Functional Annotation}

Genes were predicted in the $S$. cerevisiae strain CEN. PK113-7D genome based on the homologies to known or putative genes in the public database of the $S$. cerevisiae S288c v12.0, sequence available at the Saccharomyces Genome Database (SGD). The statistical features of the genes were predicted by a combination of genefinding software. To perform annotation, BLASTN and GlimmerHMM were applied. GlimmerHMM was used for prediction the gene structures of strain CEN.PK1137D by training the gene models from the strain S288c. All of the predicted protein-coding genes were annotated by searching against the SGD database using BLASTP, followed by manual curation.

\section{Development of Genome Browser and SNPs Viewer}

The genome browser and SNP viewer were implemented by PHP source code and MySQL database management in our hosting server http://members.dot5hosting. com/controlpanel/. Currently, our MySQL database structure contains different features that correspond to chromosome location, gene position, gene function, GO process, metabolism, and SNPs.

\section{Fermentation \\ Medium Formulation}

A chemically defined minimal medium of composition $5.0 \mathrm{~g} \mathrm{~L}^{-1}\left(\mathrm{NH}_{4}\right)_{2} \mathrm{SO}_{4}, 3.0 \mathrm{~g} \mathrm{~L}^{-1} \mathrm{KH}_{2} \mathrm{PO}_{4}, 0.5 \mathrm{~g} \mathrm{~L}^{-1}$ $\mathrm{MgSO}_{4} .7 \mathrm{H}_{2} \mathrm{O}, 1.0 \mathrm{~mL} \mathrm{~L}{ }^{-1}$ trace metal solution, $300 \mathrm{mg}$ $\mathrm{L}^{-1}$ uracil, $0.05 \mathrm{~g} \mathrm{~L}^{-1}$ antifoam 204 (Sigma-Aldrich A8311 ), and $1.0 \mathrm{~mL} \mathrm{~L}^{-1}$ vitamin solution was used for all shake flask and $2 \mathrm{~L}$ well-controlled fermentations [51]. The trace elment solution included $15 \mathrm{~g} \mathrm{~L}^{-1}$ EDTA, $0.45 \mathrm{~g} \mathrm{~L}^{-1} \mathrm{CaCl}_{2} .2 \mathrm{H}_{2} \mathrm{O}, 0.45 \mathrm{~g} \mathrm{~L}^{-1} \mathrm{ZnSO}_{4} .7 \mathrm{H}_{2} \mathrm{O}, 0.3 \mathrm{~g} \mathrm{~L}^{-1}$ $\mathrm{FeSO}_{4} \cdot 7 \mathrm{H}_{2} \mathrm{O}, 100 \mathrm{mg} \mathrm{L}^{-1} \mathrm{H}_{3} \mathrm{BO}_{4}, 1 \mathrm{~g} \mathrm{~L}^{-1} \mathrm{MnCl}_{2} \cdot 2 \mathrm{H}_{2} \mathrm{O}$, $0.3 \mathrm{~g} \mathrm{~L}^{-1} \mathrm{CoCl}_{2} \cdot 6 \mathrm{H}_{2} \mathrm{O}, 0.3 \mathrm{~g} \mathrm{~L}^{-1} \mathrm{CuSO}_{4} .5 \mathrm{H}_{2} \mathrm{O}, 0.4 \mathrm{~g} \mathrm{~L}^{-1}$ $\mathrm{NaMoO}_{4} \cdot 2 \mathrm{H}_{2} \mathrm{O}$. The $\mathrm{pH}$ of the trace metal solution was adjusted to 4.0 with $2 \mathrm{M} \mathrm{NaOH}$ and heat sterilized. The vitamin solution included $50 \mathrm{mg} \mathrm{L}^{-1} \mathrm{~d}$-biotin, $200 \mathrm{mg}$ $\mathrm{L}^{-1}$ para-amino benzoic acid, $1 \mathrm{~g} \mathrm{~L}^{-1}$ nicotinic acid, $1 \mathrm{~g} \mathrm{~L}^{-1}$ Ca.pantothenate, $1 \mathrm{~g} \mathrm{~L}^{-1}$ pyridoxine $\mathrm{HCl}, 1 \mathrm{~g} \mathrm{~L}^{-1}$ thiamine $\mathrm{HCl}$, and $25 \mathrm{mg} \mathrm{L}^{-1}$ m.inositol. The $\mathrm{pH}$ of the vitamin solution was adjusted to 6.5 with $2 \mathrm{M} \mathrm{NaOH}$, sterile-filtered and the solution was stored at $4^{\circ} \mathrm{C}$. The final formulated medium, excluding glucose and vitamin solution supplementation, is adjusted to $\mathrm{pH} 5.0$ with $2 \mathrm{M} \mathrm{NaOH}$ and heat sterilized. For carbon-limited cultivations the sterilized medium is supplemented with $40 \mathrm{~g} \mathrm{~L}^{-1}$ glucose or $40 \mathrm{~g} \mathrm{~L}^{-1}$ galactose, heat sterilized separately, and $1.0 \mathrm{~mL}$ $\mathrm{L}^{-1}$ vitamin solution is added by sterile filtration $(0.20 \mu \mathrm{m}$ pore size Ministart ${ }^{\oplus}$-Plus Sartorius AG, Goettingen, Germany).

\section{Shake Flask Cultivations and Stirred Tank Fermentations}

Shake flask cultivations were completed in $500 \mathrm{~mL}$ Erlenmeyer flasks with two diametrically opposed baffles and two side-necks with septums for sampling by syringe. Flasks were heat sterilized with $100 \mathrm{~mL}$ of medium, inoculated with a single colony, and incubated at $30^{\circ} \mathrm{C}$ with orbital shaking at 150 RPM. Stirred tank fermentations were completed in well-controlled, aerobic, 2.2L Braun Biotech Biostat B fermentation systems with a working volume of 2L (Sartorius AG, Goettingen, Germany). The temperature was controlled at $30^{\circ} \mathrm{C}$. The fermenters were outfitted with two disk-turbine impellers rotating at 600 RPM. Dissolved oxygen was monitored with an autoclavable polarographic oxygen electrode (Mettler-Toledo, Columbus, OH). During aerobic cultivation the air sparging flow rate was $2 \mathrm{vvm}$. The $\mathrm{pH}$ was kept constant at 5.0 by automatic addition of $2 \mathrm{M} \mathrm{KOH}$. Off-gas passed through a condenser to minimize the evaporation from the fermenter. The fermenters were inoculated from shake flask precultures to an initial $\mathrm{OD}_{600} 0.01$. Two biological replicates were performed for each fermentation condition.

\section{Off-gas Analysis}

The effluent fermentation gas was measured every 30 seconds for determination of $\mathrm{O}_{2}(\mathrm{~g})$ and $\mathrm{CO}_{2}(\mathrm{~g})$ concentrations by the off-gas analyzer Brüel and Kjær 1308 (Brüel \& Kjær, Nærum, Denmark).

\section{Biomass Determination}

The optical density (OD) was determined at $600 \mathrm{~nm}$ using a Shimadzu UV mini 1240 spectrophotometer (Shidmazu Europe GmbH, Duisberg, Germany). Duplicate samples were diluted with deionized water to obtain $\mathrm{OD}_{600}$ measurements in the linear range of 0-0.4 $\mathrm{OD}_{600}$ Samples were always maintained at $4{ }^{\circ} \mathrm{C}$ postsampling until $\mathrm{OD}_{600}$ and dry cell weight (DCW) measurements were performed. DCW measurements were determined through the exponential phase, until stationary phase was confirmed according to $\mathrm{OD}_{600}$ and off-gas analysis. Nitrocellulose filters $(0.45 \mu \mathrm{m}$ Sartorius AG, Goettingen, Germany) were used. The filters were predried in a microwave oven at $150 \mathrm{~W}$ for $10 \mathrm{~min}$., and cooled in a dessicator for $10 \mathrm{~min} .5 .0 \mathrm{~mL}$ of fermentation broth were filtered, followed by $10 \mathrm{~mL}$ DI water. Filters were then dried in a microwave oven for $20 \mathrm{~min}$. at $150 \mathrm{~W}$, cooled for $15 \mathrm{~min}$. in a desiccator, and the mass was determined.

\section{Metabolite Concentration Determination}

All fermentation samples were immediately filtered using a $0.45 \mu \mathrm{m}$ syringe-filter (Sartorius AG, Goettingen, 
Germany) and stored at $-20^{\circ} \mathrm{C}$ until further analysis. Glucose, ethanol, glycerol, acetate, succinate, pyruvate, fumarate, citrate, oxalate, and malate were determined by HPLC analysis using an Aminex HPX-87 $\mathrm{H}$ ionexclusion column (Bio-Rad Laboratories, Hercules, CA). The column was maintained at $65^{\circ} \mathrm{C}$ and elution performed using $5 \mathrm{mM} \mathrm{H}_{2} \mathrm{SO}_{4}$ as the mobile phase at a

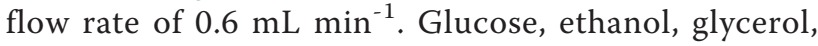
acetate, succinate, citrate, fumarate, malate, oxalate were detected on a Waters 410 differential refractometer detector (Shodex, Kawasaki, Japan), and acetate and pyruvate were detected on a Waters 468 absorbance detector set at $210 \mathrm{~nm}$. Ergosterol measurements were made according to previous published methods [52].

\section{Transcriptomics}

\section{RNA Sampling and Isolation}

Samples for RNA isolation from two biological replicates from the late-exponential phase of glucose-limited and galactose-limited batch cultivations were taken by rapidly sampling $25 \mathrm{~mL}$ of culture into a $50 \mathrm{~mL}$ sterile Falcon tube with $40 \mathrm{~mL}$ of crushed ice in order to decrease the sample temperature to below $2{ }^{\circ} \mathrm{C}$ in less than $10 \mathrm{~s}$. Cells were immediately centrifuged (4000 $\mathrm{RCF}$ at $0^{\circ} \mathrm{C}$ for $2.5 \mathrm{~min}$.), the supernatant discarded, and the pellet frozen in liquid nitrogen and it was stored at $-80^{\circ} \mathrm{C}$ until total RNA extraction. Total RNA was extracted using the FastRNA Pro RED kit (QBiogene, Carlsbad, USA) according to manufacturer's instructions after partially thawing the samples on ice. RNA sample integrity and quality was determined prior to hybridization with an Agilent 2100 Bioanalyzer and RNA 6000 Nano LabChip kit according to the manufacturer's instruction (Agilent, Santa Clara, CA).

\section{Probe Preparation and Hybridization to DNA Microarrays}

mRNA extraction, cDNA synthesis, labeling, and array hybridization to Affymetrix Yeast Genome Y2.0 arrays were performed according to the manufacturer's recommendations (Affymetrix GeneChip ${ }^{\oplus}$ Expression Analysis Technical Manual, 2005-2006 Revision 2.0). Washing and staining of arrays were performed using the GeneChip Fluidics Station 450 and scanning with the Affymetrix GeneArray Scanner (Affymetrix, Santa Clara, CA).

\section{Microarray Gene Transcription Analysis}

Affymetrix Microarray Suite v5.0 was used to generate CEL files of the scanned DNA microarrays. These CEL files were then processed using the statistical language and environment R v5.3 (R Development Core Team, 2007, http://www.r-project.org), supplemented with Bioconductor v2.3 (Biconductor Development Core Team, 2008, http://www.bioconductor.org) packages Biobase, affy, gcrma, and limma [53]. The probe intensities were normalized for background using the robust multiarray average (RMA) method only using perfect match (PM) probes after the raw image file of the DNA microarray was visually inspected for acceptable quality. Normalization was performed using the qspline method and gene expression values were calculated from PM probes with the median polish summary. Statistical analysis was applied to determine differentially expressed genes using the limma statistical package. Moderated $t$-tests between the sets of experiments were used for pair-wise comparisons. Empirical Bayesian statistics were used to moderate the standard errors within each gene and BenjaminiHochberg's method was used to adjust for multi-testing. A cut-off value of adjusted $p<0.01$ was used for statistical significance [54]. Statistically significant differential gene expression lists were then submitted to the GO Term Finder (version 0.83) of the Saccharomyces Genome Database (SGD) for GO process, function, and component statistically significant identification $(p<$ 0.01). Furthermore, the same differential gene expression lists were submitted to the Expression Viewer (Pathway Tools version 12.0 generated by SRI International on SGD) for metabolic pathway mapping and identification [11].

\section{Data Deposition}

Genome sequence and annotated gene of $S$. cerevisiae CEN.PK113-7D were deposited at sysbio database http://www.sysbio.se/cenpk. Normalized gene expression data were deposited at the GEO database http://www. ncbi.nlm.nih.gov/geo/ with accession number GPL2529 (platform), GSM536874-GSM536881 (samples), and GSE21479 (series).

\section{Additional material}

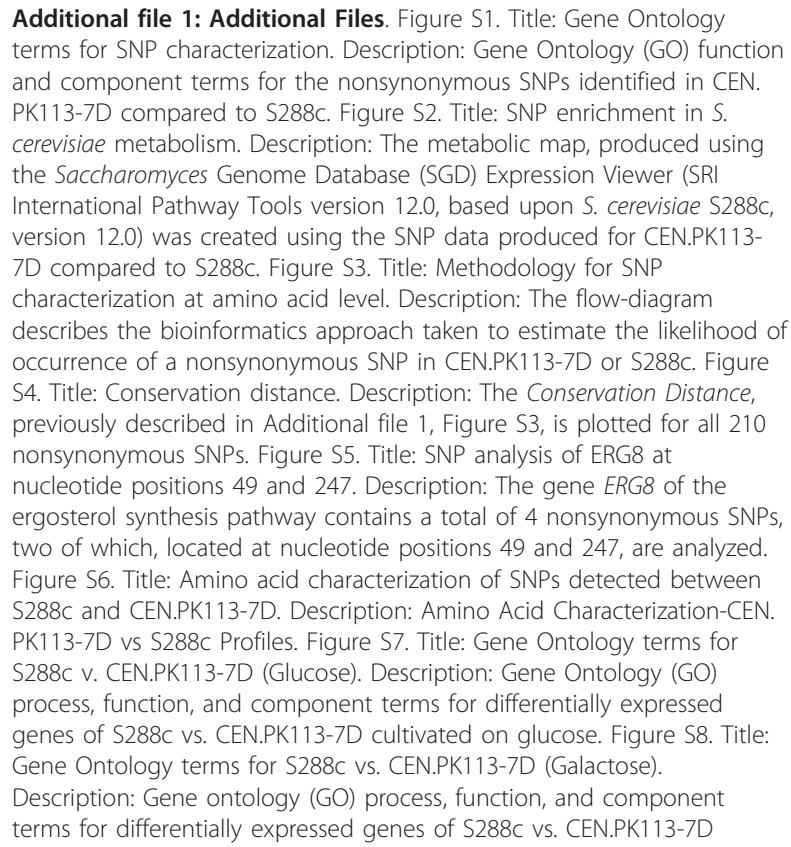


cultivated on galactose. Figure S9. Title: Metabolic pathway expression mapping for S288c Glucose vs. CEN.PK113-7D Glucose. Description: The metabolic map produced using the Saccharomyces Genome Database (SGD) Expression Viewer (SRI International Pathway Tools version 12.0, based upon S. cerevisiae S288c, version 12.0) was created using statistically significant $\log _{2}$-fold expression values for $\$ 288 \mathrm{C}$ glucose vs. CEN.PK113-7D glucose. Figure S10. Title: Metabolic pathway expression mapping for S288c Galactose vs. CEN.PK113-7D Galactose. Description: The metabolic map produced using the Saccharomyces Genome Database (SGD) Expression Viewer (SRI International Pathway Tools version 12.0, based upon S. cerevisiae S288C, version 12.0) was created using statistically significant $\log _{2}$-fold expression values for $\$ 288 \mathrm{C}$ galactose vs. CEN.PK113-7D galactose. Table S1. Title: TranscriptomeS288C v CEN.PK113-7D Glucose. Description: List of significant genes $\left(p_{a d j}<0.01\right)$. Table S2. Title: Transcriptome-S288c v CEN.PK113-7D Galactose. Description: List of significant genes ( $p_{\text {adj }}<0.01$ ). Table S3. Title: Description of $S$. cerevisiae strains. Table S4. Title: EDENA determination for de novo assembly of S288C and CEN.PK113-7D sequences

\section{Acknowledgements}

We would like to thank Merck Doctoral Fellowship for financial support to JMO and Chalmers University of Technology Foundation for financial support to W. We would like to thank Kwanjeera Wanichthanarak for supporting on database development and Gionata Scalcinati for assisting on manuscript revision.

\section{Author details}

'Department of Chemical and Biological Engineering, Chalmers University of Technology, SE-41296 Gothenburg, Sweden. ${ }^{2}$ Center for Microbial Biotechnology, Department of Systems Biology, Technical University of Denmark DK-2800, Kgs. Lyngby, Denmark. ${ }^{3}$ Fasteris SA, Geneva, Switzerland. ${ }^{4}$ Firmenich SA, Corporate Research \& Development Division, Geneva, Switzerland. ${ }^{5}$ Vaccine \& Biologics Process Development, Vaccine Research \& Development, Merck Research Labs, West Point, PA, USA. ${ }^{6}$ Biotechnology Group, Faculty of Advanced Sciences and Technologies, University of Isfahan, Isfahan 81746-73441, Iran. ${ }^{7}$ Fluxome Sciencies A/S, Research \& Development, DK-3660 Stenlose, Denmark. ${ }^{8}$ Center for Systems Biology, Soochow University, Suzhou 215006, China.

\section{Authors' contributions}

$J M O, W V, M A A, R O, J M, L F, L B, M \varnothing, M S, A C$, and JN all contributed to the conception and design of the study. JMO and MAA performed all fermentation and physiological characterization. JMO performed transcriptome measurements and analysis. $L F, L B$, and $M \varnothing$ performed highthroughput genome sequencing. JMO, WV, MAA, RO, and JM performed genome and phenotype annotation. $W$ developed the genome browser and other bioinformatics tools. JMO, WW, MAA, RO, JM, LF, MS, AC, and JN all contributed to the overall study analysis, drafting, and reviewing of the manuscript. All authors read and approved the final manuscript.

Received: 27 May 2010 Accepted: 22 December 2010 Published: 22 December 2010

\section{References}

1. Bailey JE: Toward a science of metabolic engineering. Science 1991, 252(5013):1668-1675.

2. Nielsen J: Metabolic engineering. App/ Microbiol Biotechnol 2001, 55(3):263-283

3. Stephanopoulos $G$, Vallino JJ: Network rigidity and metabolic engineering in metabolite overproduction. Science 1991, 252(5013):1675-1681.

4. Tyo KE, Alper HS, Stephanopoulos GN: Expanding the metabolic engineering toolbox: more options to engineer cells. Trends Biotechnol 2007, 25(3):132-137.

5. Warner JR, Patnaik R, Gill RT: Genomics enabled approaches in strain engineering. Curr Opin Microbiol 2009, 12(3):223-230.
6. Nielsen J, Jewett MC: Impact of systems biology on metabolic engineering of Saccharomyces cerevisiae. FEMS Yeast Res 2008, 8(1):122-131.

7. Oliver SG: From genomes to systems: the path with yeast. Philos Trans $R$ Soc Lond B Biol Sci 2006, 361(1467):477-482.

8. Westerhoff HV, Palssøn BO: The evolution of molecular biology into systems biology. Nat Biotechnol 2004, 22(10):1249-1252.

9. Förster J, Famili I, Fu P, Palssøn, Nielsen J: Genome-scale reconstruction of the Saccharomyces cerevisiae metabolic network. Genome Res 2003, 13(2):244-53.

10. Goffeau A, Barrell BG, Bussey H, Davis RW, Dujon B, Feldmann H, Galibert F, Hoheisel JD, Jacq C, Johnston M, Louis EJ, Mewes HW, Murakami Y, Philippsen P, Tettelin H, Oliver SG: Life with 6000 genes. Science 1996, 274(5287):546-563, 7.

11. Cherry JM, Ball C, Weng S, Juvik G, Schmidt R, Adler C, Dunn B, Dwight S, Riles L, Mortimer RK, Botstein D: Genetic and physical maps of Saccharomyces cerevisiae. Nature 1997, 387:67-73.

12. Herrgård MJ, Swainston N, Dobson P, Dunn WB, Arga KY, Arvas M, Blüthgen N, Borger S, Costenoble R, Heinemann M, Hucka M, Le Novère N, Li P, Liebermeister W, Mo ML, Oliveira AP, Petranovic D, Pettifer S, Simeonidis E, Smallbone K, Spasić I, Weichart D, Brent R, Broomhead DS, Westerhoff HV, Kirdar B, Penttilä M, Klipp E, Palsson BØ, Sauer U, Oliver SG, Mendes P, Nielsen J, Kell DB: A consensus yeast metabolic network reconstruction obtained from a community approach to systems biology. Nat Biotechnol 2008, 26(10):115-1160.

13. Nookaew I, Jewett MC, Meechai A, Theammarongtham C, Laoteng K, Cheevadhanarak S, Nielsen J, Bhumriatana S: The genome-scale metabolic model ilN800 of Saccharomyces cerevisiae and its validation: a scaffold to query lipid metabolism. BMC Syst Biol 2008, 2:71.

14. Nielsen J: Physiological engineering aspects of Penicillium chrysogenum. Polyteknisk Forlag, Lyngby 1995, 13.

15. Kahvejian A, Quanckenbush J, Thompson JF: What would you do if you could sequence everything? Nat Biotechnol 2008, 26(10):1125-1133.

16. Morozova O, Marra MA: Applications of next-generation sequencing technologies in functional genomics. Genomics 2008, 92:255-264.

17. Shendure J, Ji H: Next-generation DNA sequencing. Nat Biotechnol 2008, 26(10):1135-1145.

18. Srivatsan A, Han Y, Peng J, Tehranchi AK, Gibbs R, Wang JD, Chen R: Highprecision, whole-genome sequencing of laboratory strains facilitates genetic studies. PLOS Genetics 2008, 4(8):e1000139.

19. Schacherer J, Ruderfer DM, Gresham D, Dolinski K, Botstein D, Kruglyak : Genome-wide analysis of nucleotide-level variation in commonly used Saccharomyces cerevisiae strains. PLOS One 2007, 3:e322.

20. Carter D: Saccharomyces Genome Resequencing Project: User Manual. [http://www.sanger.ac.uk/Teams/Team118//sgrp/], Last accessed September 4, 2009 (Original publication: 2008).

21. Hernandez D, François $P$, Farinelli L, Osterås $M$, Schrenzel J: De novo bacterial genome sequencing: millions of very short reads assembled on a desktop computer. Genome Res 2008, 18(5):802-809.

22. Nikolaev SI, Iseli C, Sharp AJ, Robyr D, Rougemont J, Gehrig C, Farinelli L, Antonarakis SE: Detection of genomic variation by selection of a $9 \mathrm{Mb}$ DNA region and high throughput sequencing. PLOS One 2009, 4(8):e6659.

23. Rougemonet J, Amzallag A, Iseli C, Farinelli L, Xenarios I, Naef F: Probabilistic case calling of Solexa sequencing data. BMC Bioinformatics 2008, 9:431.

24. van Dijken JP, Bauer J, Brambilla L, Duboc P, François JM, Gancedo C, Giuseppin ML, Heijnen JJ, Hoare M, Lange HC, Madden EA, Niederberger P, Nielsen J, Parrou JL, Petit T, Porro D, Reuss M, van Riel M, Rizzi M, Steensma HY, Verrips CT, Vindeløv J, Pronk JT: An interlaboratory comparison of physiological and genetic properties of four Saccharomyces cerevisiae strains. Enzyme Microb Technol 2000, 26(910):706-714.

25. Monteiro PT, Mendes N, Teixiera MC, d-Orey S, Tenreiro S, Mira N, Pais H, Francisco AP, Carvalho AM, Lourenço A, Sá-Correia I, Oliveria AL, Freitas AT: YEASTRACT-DICOVERER: new tools to improve the analysis of transcriptional regulatory association in Saccharomyces cerevisiae. Nucl Acids Res 2008, 36:D132-D136.

26. Teixeria MC, Monteiro $P$, Jain $P$, Tenreiro $S$, Fernandes AR, Mira NP, Alenquer M, Freitas AT, Oliveira AL, Sá-Correia I: The YEASTRACT database: a tool for the analysis of transcription regulatory associations in Saccharomyces cerevisiae. Nucl Acids Res 2006, 34:D446-D451. 
27. Douglas LM, Li L, Yang Y, Dranginis AM: Expression and characterization of the flocculin Flo11/Muc1, a Saccharomyces cerevisiae mannoprotein with homotypic properties of adhesion. Eukaryot Cell 2007, 6(12):2214-2221

28. McCammon MT, Epstein CB, Przybyla-Zawislak B, McAlister-Henn L, Butow RA: Global transcription analysis of Krebs tricarboxylic acid cycle mutants reveals an alternating pattern of gene expression and effects of hypoxic and oxidative genes. Mol Biol Cell 2003, 14(3):958-972.

29. Daum G, Tuller G, Nemec T, Hrastnik C, Balliano G, Cattel L, Milla P, Rocco F, Conzelmann A, Vionnet C, Kelly DE, Kelly S, Schweizer E, Schüller H-J, Hojad U, Greiner E, Finger K: Systematic analysis of yeast strains with possible defects in lipid metabolism. Yeast 1999, 15(7):601-614.

30. Winston F, Dollard C, Ricupero-Hovasse SL: Construction of a set of convenient Saccharomyces cerevisiae strains that are isogenic to S288c. Yeast 1995, 11(1):53-55.

31. Jennings SM, Tsay TH, Fisch TM, Robinson GW: Molecular cloning and characterization of the yeast gene for squalene synthesis. Proc Natl Acad Sci USA 1991, 88:6038-6042.

32. Tsay YH, Robinson GW: Cloning and characterization of ERG8, an essential gene of Saccharomyces cerevisiae that encodes phosphomevalonate kinase. Mol Cell Biol 1991, 11(2):620-631.

33. Basson ME, Thorsness M, Finer-Moore J, Stroud RM, Rine J: Structural and functional conservation between yeast and human 3-hydroxy-3methglutaryl coenzyme A reductases, the rate-limiting enzyme of sterol biosynthesis. Mol Cell Biol 1988, 8(9):3797-3808.

34. Maury J, Asadollahi MA, Møller K, Clark A, Nielsen J: Microbial isoprenoid production: an example of green chemistry through metabolic engineering. Adv Biochem Engin/Biotechnol 2005, 100:19-51.

35. Grabowska D, Karst F, Szkopinska A: Effect of squalene synthase gene disruption on synthesis of polyprenols in Saccharomyces cerevisiae. FEBS Lett 1998, 434(3):406-408.

36. Basson ME, Thorsness M, Rine J: Saccharomyces cerevisiae contains two functional genes encoding 3-hydroxy-3-methylglutaryl-coenzyme A reductase. Proc Natl Acad Sci USA 1986, 83(15):5563-5567.

37. Bloch K, Chaykin S, Phillips AH, de Waard A: Mevalonic acid pyrophosphate and isopentenylpyrophosphate. J Biol Chem 1959, 234:2595-2604.

38. Durr IF, Rudney H: The reduction of beta-hydroxy-beta-methyl-glutaryl coenzyme A to mevalonic acid. J Biol Chem 1960, 235:2572-2578.

39. Eberhardt NL, Rilling HC: Prenyltransferase from Saccharomyces cerevisiae. Purification to homogeneity and molecular properties. J Biol Chem 1975 250(3):863-866.

40. Gray JC, Kekwick RG: The inhibition of plant mevalonate kinase preparations by prenyl pyrophosphates. Biochim Biophys Acta 1972, 279(2):290-296.

41. Middleton B, Tubbs PK: 3-Hydroxy-3-methylglutaryl-CoA synthase from baker's yeast. Methods Enzymol 1975, 35:173-177.

42. Porter JW: Mevalonate kinase. Methods Enzymol 1985, 110:71-78.

43. Rilling HC: Eukaryotic prenyltransferases. Methods Enzymol 1985 110:145-152.

44. Tchen TT: Mevalonic kinase: purification and properties. J Biol Chem 1958, 233(5):1100-1103.

45. Bhat PJ, Hopper JE: Overproduction of the GAL1 or GAL3 protein causes galactose-independent activation of the GAL4 protein: evidence for a new model of induction for the yeast GAL/MEL region. Mol Cell Biol 1992, 12(6):2701-2707

46. Bhat PJ, Oh D, Hopper JE: Analysis of the GAL3 signal transduction pathway activating GAL4 protein-dependent transcription in Saccharomyces cerevisiae. Genetics 1990, 125(2):281-291.

47. Douglas HC, Hawthorne DC: Enzymatic expression and genetic linkage of genes controlling galactose utilization in Saccharomyces. Genetics 1964, 49:837-844.

48. Clausen M, Lamb CJ, Megnet R, Doerner PW: PAD1 encodes phenylacrylic acid decarboxylase which confers resistance to cinnamic acid in Saccharomyces cerevisiae. Gene 1994, 142(1):107-112

49. Mortimer RK, Johnston JR: Genealogy of principal strains of the yeast genetic stock center. Genetics 1986, 113(1):35-43.

50. Johnston M, Andrew S, Brinkman R, Cooper J, Ding H, Dover J, Favello A, Fulton L, Gattung S, Geisel C, Kirsten J, Kucaba T, Hillier L, Jier M, Johnston L, Langston Y, Latreille P, Louis EJ, Macri C, Mardis E, Menezes S, Mouser L, Nhan M, Rifkin L, Riles L, Peter HS, Trevaskis E, Vaughan K,
Vignati D, Wilcox L, Wohldman P, Waterston R, Wilson R, Vaudin M: Complete nucleotide sequence of Saccharomyces cerevisiae chromosome VIII. Science 1994, 265(5181):2077-82.

51. Verudyn C, Postma E, Scheffers WA, Van Dijken JP: Effect of benzoic acid on metabolic fluxes in yeasts: a continuous culture study on the regulation of respiration and alcoholic fermentation. Yeast 1992, 8:501-517.

52. Asadollahi MA, Maury J, Møller K, Nielsen KF, Schalk M, Clark A, Nielsen J: Production of plant sesquiterpenes in Saccharomyces cerevisiae: effect of ERG9 repression on sesquiterpene biosynthesis. Biotechnol Bioeng 2008, 99(3):666-677.

53. Smyth GK: Limma: linear models for microarray data. In Bioinformatics and Computational Biology Solutions using $R$ and Bioconductor. Edited by: Gentleman R, Carey V, Dudoit S, Irizarry R, Huber W. Springer, New York; 2005:397-420.

54. Smyth GK: Linear models and empirical Bayes methods for assessing differential expression in microarray experiments. Statistical Applications in Genetics and Molecular Biology 2000, 3, 1(3).

doi:10.1186/1471-2164-11-723

Cite this article as: Otero et al:: Whole genome sequencing of Saccharomyces cerevisiae: from genotype to phenotype for improved metabolic engineering applications. BMC Genomics 2010 11:723.

\section{Submit your next manuscript to BioMed Central and take full advantage of:}

- Convenient online submission

- Thorough peer review

- No space constraints or color figure charges

- Immediate publication on acceptance

- Inclusion in PubMed, CAS, Scopus and Google Scholar

- Research which is freely available for redistribution

Submit your manuscript at www.biomedcentral.com/submit
C Biomed Central 